\title{
Efficient numerical solution of acoustic scattering from doubly-periodic arrays of axisymmetric objects
}

\author{
Yuxiang Liu*,b, Alex H. Barnett ${ }^{\mathrm{a}, \mathrm{b}}$ \\ ${ }^{a}$ Department of Mathematics, Dartmouth College, Hanover, NH 03755, USA \\ ${ }^{b}$ Department of Physics $\mathcal{G}$ Astronomy, Dartmouth College, Hanover, NH 03755, USA
}

\begin{abstract}
We present a high-order accurate boundary-based solver for three-dimensional (3D) frequency-domain scattering from a doubly-periodic grating of smooth axisymmetric sound-hard or transmission obstacles. We build the one-obstacle solution operator using separation into $P$ azimuthal modes via the FFT, the method of fundamental solutions (with $N$ proxy points lying on a curve), and dense direct least-squares solves; the effort is $O\left(N^{3} P\right)$ with a small constant. Periodizing then combines fast multipole summation of nearest neighbors with an auxiliary global Helmholtz basis expansion to represent the distant contributions, and enforcing quasiperiodicity and radiation conditions on the unit cell walls. Eliminating the auxiliary coefficients, and preconditioning with the one-obstacle solution operator, leaves a well-conditioned square linear system that is solved iteratively. The solution time per incident wave is then $O(N P)$ at fixed frequency. Our scheme avoids singular quadratures, periodic Green's functions, and lattice sums, and its convergence rate is unaffected by resonances within obstacles. We include numerical examples such as scattering from a grating of period $13 \lambda \times 13 \lambda$ comprising highly-resonant sound-hard "cups" each needing $N P=64800$ surface unknowns, to 10-digit accuracy, in half an hour on a desktop.
\end{abstract}

Key words: scattering, Helmholtz, acoustic, diffraction, grating, meta-materials, periodic, fundamental solutions 2010 MSC: $65 \mathrm{~N} 38,65 \mathrm{~N} 80$

\section{Introduction}

The control of waves using periodic structures is crucial for modern optical, electromagnetic and acoustic devices such as diffraction gratings, filters, photonic crystals and meta-materials [45], solar cells [4], and absorbers [64, 27]. Periodic scattering problems also arise in monitoring [63] or imaging [61] a patterned structure. Outside of asymptotic regimes where analytic models are useful, efficient and accurate numerical simulation is key to assess sensitivity to changes in parameters, and to optimize those parameters to improve device performance.

Here we present a solver for 3D acoustic scattering from a doubly-infinite array of isolated axisymmetric objects, as shown in Fig. 1(a). With acoustic applications in mind, we focus on the Neumann (sound-hard) boundary condition (including resonators) and transmission problems, and on highly accurate solutions. Sound absorbing surfaces often involve periodic structures such as perforated slabs [64, Sec. 6.5.4], resonators [64, Sec. 9.2.3] [29, Ch. 12], or wedges (as in anechoic chamber walls) [29, Fig. 12-13]. Recently, there has also been interest in acoustic meta-materials [25] or "phononics", including new phenomena such as anomalous transmission [25, Ch. 4] and acoustic cloaking [75]. The new techniques we present are relatively simple to extend to multilayer geometries (such as perforated slabs) and poroelastic media (such as foams) common in noise control [65]. We view this work-in particular the periodizing scheme, which is very general-as a step toward 3D multilayer periodic boundary-based solvers (generalizing recent work in 2D [21]) for acoustics, coupled acoustics-elastodynamics, the Maxwell equations, and Stokes flow.

Let us explain where our contribution fits into the bigger picture. Direct volume discretization methods including the finite element $[6,63]$ and finite difference time-domain $[71,40]$ are common for acoustic scattering problems, and

*Corresponding author. Tel.: +1 6032770791.

Email address: Yuxiang. Liu.GR@dartmouth.edu (Yuxiang Liu)

Preprint submitted to Journal of Computational Physics

June 7, 2016 
can be successful for low-to-medium frequencies and accuracies. However, low-order finite elements suffer from the accumulation of phase errors across the domain (known as "pollution" [5]), meaning that an increasing number of unknowns per wavelength are needed as the wavenumber $k$ grows, so that greater than $O\left(k^{3}\right)$ unknowns are needed to maintain accuracy. High-order finite elements, while showing recent promise for medium-frequency acoustics at accuracies of a couple of digits [11], are tricky to generate in complex geometries. Time-domain methods (e.g. FDTD) also suffer from a low convergence order (usually at most 1st-order in the presence of surfaces; recent work has recovered 2nd-order [40]), difficulty in modeling impedance boundary conditions [32], and very long settling times if a structure is resonant. It should be noted that via the Fourier transform, FDTD can solve many frequencies in a single shot. However, accuracies from such methods are commonly 1-2 digits, even with dozens of grid points per wavelength [40].

For piecewise-uniform media, boundary-based methods become more efficient than direct discretization once the geometry is more than a couple of wavelengths across, and/or if an accuracy beyond a couple of digits is needed. Only $\mathcal{N}=O\left(k^{2}\right)$ unknowns are needed for a smooth obstacle if a high-order surface quadrature is used. The most common approach is the boundary integral method (BIE), in which the scattered wave is represented via potential theory using the Helmholtz Green's function

$$
G_{k}(\mathbf{x}, \mathbf{y}):=\frac{e^{i k|\mathbf{x}-\mathbf{y}|}}{4 \pi|\mathbf{x}-\mathbf{y}|}, \quad \mathbf{x}, \mathbf{y} \in \mathbb{R}^{3} .
$$

For the mathematical foundation of this method in the non-periodic setting see [22, 23], and in our periodic setting [2]. Formulation as a 2nd kind Fredholm integral equation on $\partial \Omega$, the boundary of an obstacle $\Omega \subset \mathbb{R}^{3}$, has the advantage that the condition number of the discretized linear system remains small independent of $\mathcal{N}$ as $k$ is held fixed. Using the fast multipole method (FMM) [37, 20] to apply the dense matrix discretization of the operator inside an iterative Krylov method solver such as GMRES [68] can create an $O(\mathcal{N})$ solver (at moderate frequencies). However, in practice two problems plague this otherwise attractive scheme: 1) for resonant or geometrically complex objects the large number of eigenvalues close to the origin causes the number of iterations to be unreasonably large, and direct solvers can be orders of magnitude faster [33]. 2) high-order surface quadratures in 3D are quite challenging and are still an area of active research $[17,73,66,15,10]$. We note that low-order Galerkin methods are the most commonly used in Helmholtz problems [67]. The method we propose fixes both of these problems in the axisymmetric setting.

We use a close relative of the BIE, the method of fundamental solutions (MFS, also known as auxiliary sources [70] or the charge simulation method [52]), in which (for sound-hard scattering) the scattered wave has a representation

$$
u(\mathbf{x}) \approx \sum_{j=1}^{\mathcal{N}} c_{j} G_{k}\left(\mathbf{x}, \mathbf{y}_{j}\right)
$$

where $\mathbf{y}_{j} \in \mathbb{R}^{3}, j=1, \ldots, \mathcal{N}$, are $\mathcal{N}$ source points covering a source surface $\Gamma_{-}$lying inside the obstacle $\Omega$ but close to its boundary $\partial \Omega$. The coefficients $\left\{c_{j}\right\}$ are the solution to a linear system set up by matching (2) to the boundary data at collocation points on $\partial \Omega$. The MFS idea (see the review [31]) is old, being first proposed by Kupradze-Aleksidze [55], and is common in the engineering community [28]. The MFS may be viewed as an exponentially ill-conditioned firstkind BIE. Yet, when combined with a backward stable linear solver it can achieve close to machine precision when the source points are chosen correctly [7]. An optimal choice of source points remains one of the more ad-hoc aspects of the MFS, although we demonstrate in Sec. 3.2 an excellent scheme for analytic boundaries that requires only a single adjustable parameter. The MFS has a significant advantage over BIE: because $\Gamma_{-}$is separated from $\partial \Omega$, no singular surface quadratures are needed (either for the self-interaction of the object or for evaluation of $u$ close to $\partial \Omega$ ). This will enable us to handle Neumann and transmission conditions simply (in contrast, the robust BIE formulation in the Neumann case requires handling the derivative of the double-layer operator either as a hypersingular operator [53] or using Calderón regularization [22, Sec. 3.6] [1], and in the transmission case the compact difference of such operators [22, Sec. 3.8]). The MFS has proven useful in 2D acoustic settings [65].

In the axisymmetric case the MFS becomes more efficient $[50,70,12,19]$, because the problem separates into $P$ angular Fourier modes that may be solved independently, each with a small number of unknowns $N$, the total number of unknowns being $\mathcal{N}=N P$. As we will show, many smooth obstacles up to $10 \lambda$ in diameter need only $N<10^{3}$ for 10-digit accuracy, with $P$ of order one hundred. Since dense least-squares solves of such a size $N$ are cheap, the good conditioning of a BIE approach confers little advantage over using MFS, at least for smooth domains. (Recent work 
also shows that several digits of accuracy is possible with the MFS in corner domains [44, 60].) We note that recently some technical challenges of high-order BIE on axisymmetric surfaces have been solved [74, 41, 42, 43], but not in the case of transmission boundary conditions that we address.

We now outline how we turn a scheme for solving the scattering from one obstacle into a scheme for a bi-infinite array of obstacles-we refer to this as "periodizing"; it is one of the main contributions of this paper. The standard way to periodize in $2 \mathrm{D}[62,16]$ or $3 \mathrm{D}[66,2,18]$ is to replace the free-space Green's function (1) by its quasiperiodic version which sums over all source points,

$$
G_{k}^{\mathrm{QP}}(\mathbf{x}, \mathbf{y}):=\sum_{n, m \in \mathbb{Z}} \alpha^{m} \beta^{n} G_{k}\left(\mathbf{x}, \mathbf{y}+m \mathbf{e}_{x}+n \mathbf{e}_{y}\right),
$$

where the Bloch phases are $\alpha$ and $\beta$ (defined below in (7)), and the array lattice vectors are $\mathbf{e}_{x}$ and $\mathbf{e}_{y}$ as in Fig. 1(a). The above sum is notoriously slowly convergent, hence a host of schemes such as Ewald's method [30, 46, 2, 3], other spatial-spectral splittings [47], or lattice sums [67, 58] have been developed for numerical evaluation. These schemes are generally quite complicated, both analytically and in terms of implementation, and raise two major problems:

1. While they are able to fill the $\mathcal{N}^{2}$ elements of a dense matrix, most such schemes are incompatible with the FMM or other fast algorithms. Exceptions are the lattice-sum based correction to the FMM of Otani et al [67] and the rolled-off spatial sum of Bruno et al [18].

2. At certain sets of parameters $(\alpha, \beta)$ and $k$ called Wood anomalies, the quasiperiodic Green's function does not exist, i.e. the sum (3) diverges, even though the solution to the diffraction problem remains well-posed and finite.

We propose a simple new approach, following $[9,34,21,10]$, which cures the first problem. In the setting of continuous interfaces such as [21], our approach would also cure the second; however, since we focus on isolated obstacles, then (as with the work in $[67,18]$ ) we do not attempt to address Wood anomalies here. Our representation restricts the sum (3) to only the $3 \times 3$ block of nearest neighbors, adding an auxiliary spherical harmonic basis (of maximum degree $p$ ) for smooth Helmholtz solutions in the neighborhood of the object,

$$
u(\mathbf{x}) \approx \sum_{j=1}^{\mathcal{N}} c_{j} G_{k}^{\text {near }}\left(\mathbf{x}, \mathbf{y}_{j}\right)+\sum_{l=0}^{p} \sum_{m=-l}^{l} d_{l m} j_{l}(k r) Y_{l m}(\theta, \phi), \quad \text { where } \quad G_{k}^{\text {near }}(\mathbf{x}, \mathbf{y}):=\sum_{|m|,|n| \leq 1} \alpha^{m} \beta^{n} G_{k}\left(\mathbf{x}, \mathbf{y}+m \mathbf{e}_{x}+n \mathbf{e}_{y}\right) .
$$

Here $(r, \theta, \phi)$ are spherical coordinates. The auxiliary basis represents the contribution from the remainder of the lattice of images; since they are far from $\Omega$, this has rapid exponential convergence with $p$. An expanded linear system is used to solve for the coefficients. Being rectangular and ill-conditioned (as with the plain MFS method), this cannot be solved iteratively in the case of large $\mathcal{N}$. In Sec. 4.2 we show that, because of axial symmetry, preconditioning is possible using a direct factorization of the MFS matrix pseudoinverse for the single obstacle. In the iterative scheme, the contributions from the near images are applied using the FMM, to give a scheme that solves each new incident wave in $O(\mathcal{N})$. Our work can thus be seen as a periodic generalization of the fast multibody scattering work of Gumerov-Duraiswami for spheres [38], of Gimbutas-Greengard for smooth scatterers [36], and of Hao-MartinssonYoung [41] for axisymmetric scatterers.

One novel aspect is the high accuracy we achieve (around 10 digits) compared to most other periodic integral equation work $[67,18]$. This is true even for resonant obstacles, thanks to the direct solve used for the isolated obstacle. Our scheme is very practical for periods up to a dozen wavelengths in each direction, but cannot go much higher than this in reasonable CPU time due to the $O\left(p^{6}\right)$ scaling of the dense matrix operations. However, this covers the vast majority of diffraction applications, where typically the period is of order the wavelength, and also allows "super-cell" simulations, for instance for random media.

A similar scheme has recently been proposed by Gumerov-Duraiswami [39] for periodizing the 3D Laplace equation, using proxy sources instead of the auxiliary basis in (4); however, in Appendix A we show that for our application to the 3D Helmholtz equation spherical harmonics are much more efficient.

Our paper is organized as follows. In Sec. 2 we state the two periodic scattering boundary value problems under study, namely Neumann and transmission conditions. In Sec. 3 we explain the MFS in the axisymmetric one-obstacle setting, including the choice of source points, and study its convergence when using a dense direct solve for each 

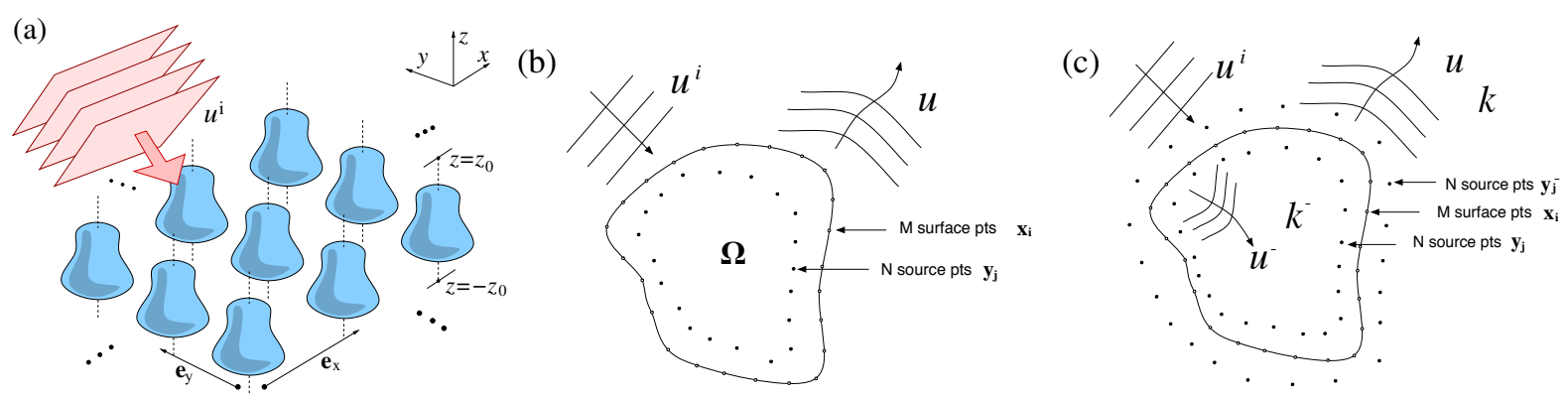

Figure 1: (a) Periodic scattering geometry in 3D. (b) Sketch of the method of fundamental solutions (MFS) for solution of an exterior BVP. (For simplicity, the 2D case is shown.) (c) MFS for a transmission problem (again, the 2D case is shown).

Fourier mode. In Sec. 4 we present the periodizing scheme and the resulting full linear system, and then show how Schur complements can turn this into a well-conditioned square system that can be solved iteratively. Numerical results are presented in Sec. 5, then we conclude in Sec. 6. The appendix compares the efficiency of our periodizing scheme with a variant using proxy points.

\section{Formulation of the boundary value problems}

Let $\Omega \subset \mathbb{R}^{3}$ be an axisymmetric obstacle with boundary $\partial \Omega$ defined by the rotation about the $z$-axis of a smooth curve $\gamma$ lying in the $\rho-z$ plane, where $(\rho, \theta, z)$ define a cylindrical coordinate system. For simplicity we consider a rectangular lattice defined by vectors $\mathbf{e}_{x}=\left(e_{x}, 0,0\right)$ and $\mathbf{e}_{y}=\left(0, e_{y}, 0\right)$, so that the grating of objects (see Fig. 1(a)) is defined by $\Omega_{\Lambda}:=\left\{(x, y, z) \in \mathbb{R}^{3}:\left(x+m e_{x}, y+n e_{y}, z\right) \in \Omega, m, n \in \mathbb{Z}\right\}$, and is assumed not to self-intersect. Note that our scheme will also apply to general (possibly skew) lattices and a general (fixed) axis of symmetry of the objects with minor changes in bookkeepping. An incident plane wave (representing pressure variation of a time-harmonic acoustic wave),

$$
u^{\mathrm{i}}(\mathbf{x})=e^{i \mathbf{k} \cdot \mathbf{x}}, \quad \mathbf{x}:=(x, y, z) \in \mathbb{R}^{3}
$$

impinges on this lattice, with given wavevector $\mathbf{k}=\left(k_{x}, k_{y}, k_{z}\right)$ whose free-space wavenumber is $k:=|\mathbf{k}|$. This incident wave is quasiperiodic in the following sense.

Definition 1. A function $u: \mathbb{R}^{3} \rightarrow \mathbb{C}$ is said to be quasiperiodic with Bloch phases $\alpha$ and $\beta$, if

$$
\alpha^{-1} u\left(x+e_{x}, y, z\right)=\beta^{-1} u\left(x, y+e_{y}, z\right)=u(x, y, z) \quad \forall(x, y, z) \in \mathbb{R}^{3} .
$$

The incident wave parameters fix the Bloch phases

$$
\alpha=e^{i \mathbf{k} \cdot \mathbf{e}_{x}}, \quad \beta=e^{i \mathbf{k} \cdot \mathbf{e}_{x}}
$$

with $|\alpha|=|\beta|=1$, and $u^{i}$ is quasiperiodic with these phases. The resulting scattered wave $u$ will share this quasiperiodic symmetry. As usual in scattering theory [23], the physical wave outside the lattice of objects is the total $u^{\mathrm{t}}=u^{\mathrm{i}}+u$. The scattered wave obeys the exterior Helmholtz equation

$$
\Delta u+k^{2} u=0, \quad \text { in } \mathbb{R}^{3} \backslash \overline{\Omega_{\Lambda}}
$$

and the upwards and downwards Rayleigh-Bloch radiation conditions [13, 69]

$$
\begin{array}{ll}
u(x, y, z)=\sum_{m, n \in \mathbf{Z}} a_{m n} \exp i\left[\kappa_{x}^{m} x+\kappa_{y}^{n} y+\kappa_{z}^{(m, n)}\left(z-z_{0}\right)\right], & z \geq z_{0},(x, y) \in \mathbb{R}^{2} \\
u(x, y, z)=\sum_{m, n \in \mathbf{Z}} b_{m n} \exp i\left[\kappa_{x}^{m} x+\kappa_{y}^{n} y+\kappa_{z}^{(m, n)}\left(-z-z_{0}\right)\right], & z \leq-z_{0},(x, y) \in \mathbb{R}^{2}
\end{array}
$$


where $z_{0}$ is such that $\bar{\Omega}$ lies between the planes $z= \pm z_{0}$, and where $\kappa_{x}^{m}:=k_{x}+2 \pi m / e_{x}, \kappa_{y}^{n}:=k_{y}+2 \pi n / e_{y}$ and $\kappa_{z}^{(m, n)}:=+\sqrt{k^{2}-\left(\kappa_{x}^{m}\right)^{2}-\left(\kappa_{y}^{n}\right)^{2}}$. define the plane wave wavevectors $\left(\kappa_{x}^{m}, \kappa_{y}^{n}, \pm \kappa_{z}^{(m, n)}\right)$. Note that the sign of the squareroot is taken as positive real or positive imaginary. These conditions state that $u$ can be written as a uniformly convergent expansion of quasiperiodic plane waves of outgoing or decaying type away from the lattice. In applications the far field amplitudes $a_{m n}$ and $b_{m n}$ are the desired quantities, giving the radiated strengths in the various Bragg orders.

We will solve two cases of obstacle scattering: 1) Neumann (sound hard) boundary conditions scattering from an impenetrable obstacle,

$$
\frac{\partial u}{\partial n}=-\frac{\partial u^{\mathrm{i}}}{\partial n}, \quad \text { on } \partial \Omega
$$

and 2) Transmission conditions, with a new wavenumber $k_{-}$and quasiperiodic scattered wave $u^{-}$inside the object (the incident wave $u^{i}$ being defined as zero inside $\Omega$ ), i.e.,

$$
\begin{aligned}
\Delta u^{-}+k_{-}{ }^{2} u^{-} & =0, \quad \text { in } \Omega \\
u-u^{-} & =-u^{\mathrm{i}} \quad \text { on } \partial \Omega \\
\frac{\partial u}{\partial n}-\frac{\partial u^{-}}{\partial n} & =-\frac{\partial u^{\mathrm{i}}}{\partial n} \quad \text { on } \partial \Omega .
\end{aligned}
$$

For simplicity, (11c) models the case where the interior and exterior densities are equal (a density difference would result in prefactors here [23, Sec. 2.1]). Note that, due to quasiperiodicity, the above PDE and boundary conditions need only be defined on a single copy of the object in the lattice.

Given an incident wave $u^{i}$ with wavevector $\mathbf{k}$, the full boundary value problem (BVP) is defined by (6)-(8), radiation conditions (9a)-(9b), and either Neumann condition (10) or transmission conditions (11a)-(11c). The following rigorous results are known about the BVPs. With a general smooth obstacle shape $\Omega$ and fixed wavespeed ratio (material property) $k_{-} / k$, the transmission BVP has a solution for all incident wave directions and frequencies $k>0$ [69, Thm. 9]. The solution is unique for all but possibly a discrete set of frequencies, for each incident wave direction [69, Thm. 8]. Such frequencies are referred to as bound states of the grating [69], and correspond to physical resonances of the BVP. Similar statements hold in the Neumann case. In the transmission case, if $k_{-}<k$ and all lines parallel to the $z$-axis intersect $\partial \Omega$ at only two points, then no such bound states can exist [69, Thm. 13]. A similar analysis for a connected interface (or multiple such interfaces) is carried out by Arens [2].

If $\kappa_{z}^{(m, n)}=0$ for any pair of integers $(m, n)$, this defines a Wood anomaly [59, 69], where one (or more) of the Rayleigh-Bloch waves is constant (non-decaying) in the $z$ direction. Although (3) does not exist at Wood anomalies (it diverges like an inverse square-root with respect to the incident wave parameters), the BVP remains well-behaved, i.e. well conditioned with respect to varying the amplitude of $u^{i}$.

Remark 1. Because a Bragg scattering mode is "on the cusp of existence" at a Wood anomaly, the rate of change of scattering coefficients $a_{m n}$ and $b_{m n}$ with respect to incident angle or frequency $k$ diverges there (as an inverse square root singularity). Thus, for example, at a parameter distance $10^{-10}$ from a Wood anomaly, we expect to lose around 5 digits of accuracy purely due to round-off in the representation of the input parameters.

In this work, we assume that we are not at a Wood anomaly. However, we will find that the method we present in Sec. 4 loses of order the same number of digits as discussed in the above remark.

\section{Method of fundamental solutions in the axisymmetric setting}

In this section we present the MFS for scattering from an isolated axisymmetric obstacle, which will form a key part of the periodic solver. We present the Neumann case first, and then explain how the transmission case differs. Thus we care to solve the BVP given by (8) in $\mathbb{R}^{3} \backslash \bar{\Omega}$, boundary conditions (10), and the usual 3D Sommerfeld radiation condition,

$$
\frac{\partial u}{\partial r}-i k u=o\left(r^{-1}\right), \quad r:=|\mathbf{x}| \rightarrow \infty
$$

where the convergence implied by the little " $o$ " is uniform in angle. We exploit standard separation of variables to represent the incident $u^{i}$ and scattered wave solution $u$ as a sum of azimuthal Fourier modes. Each mode results in an independent linear system. 

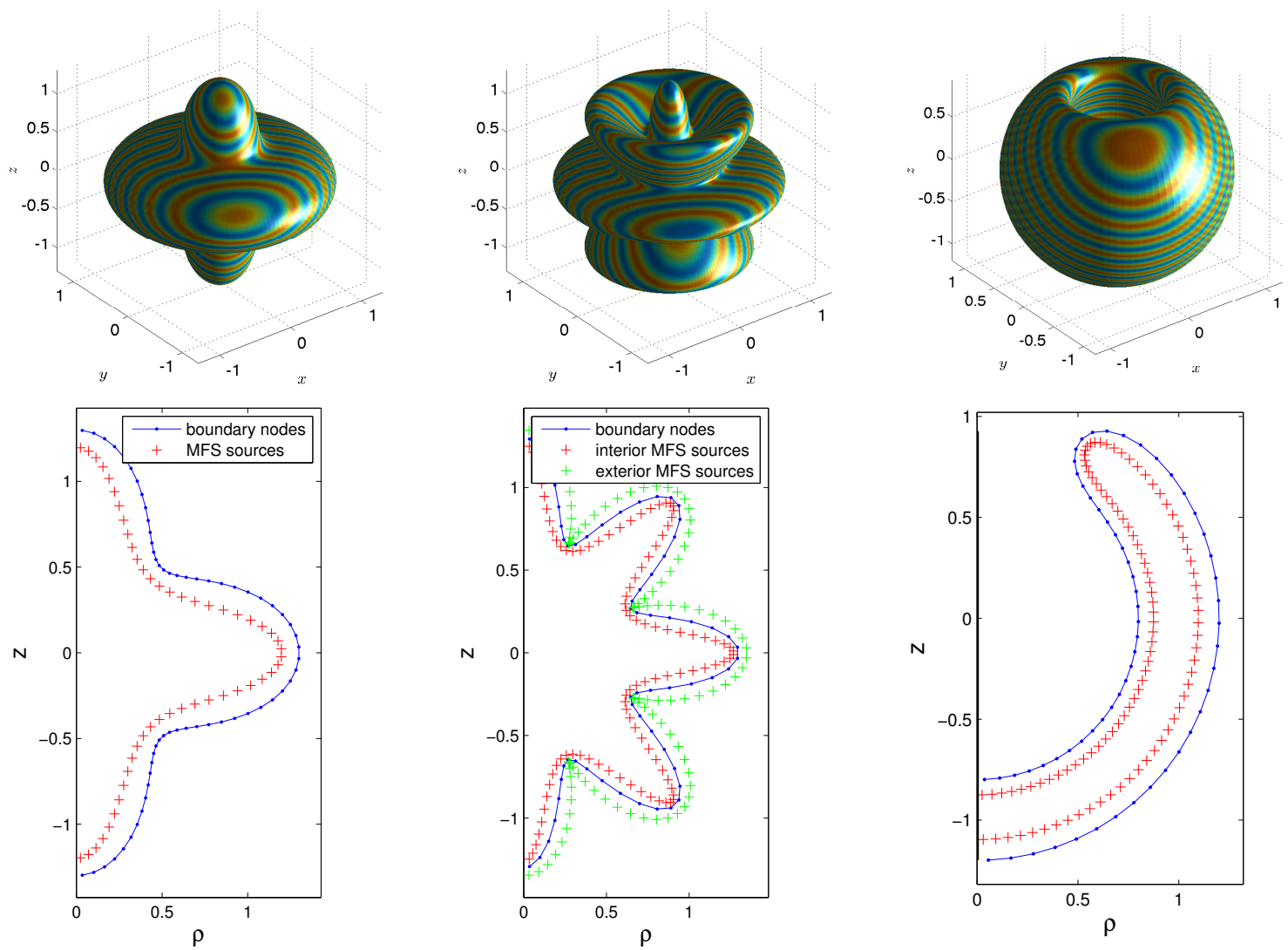

Figure 2: Obstacle bodies of revolution tested in this work. Above is the 3D surface, and below the corresponding 2D generating curve and $N$ MFS points (with $M \approx 1.2 N$ boundary points). Color above shows the real part of the incident wave $u^{\mathrm{i}}$ at the highest frequency tested $k=40 \mathrm{in} \operatorname{Sec} .3 .3$, restricted to the surface. From left to right: (a) "Smooth" shape given in polar coordinates in the $\rho$ - $z$ plane by $r(\theta)=1+0.3 \cos 4 \theta$. The $N=50$ MFS sources are shown with distance parameter is $\tau=0.1$. (b) "Wiggly" shape $r(\theta)=1+0.3 \cos 8 \theta . \tau= \pm 0.03$. $N=100$. (c) "Cup" shape given parametrically in $0 \leq t \leq \pi$ by $r(t)=1-a \operatorname{erf}[(t-\pi / 2) / a], \theta(t)=b-a+2\left(1-\frac{b-a}{\pi}\right) s_{a}(t-\pi / 2)$ where the half-thickness is $a=0.2$ and opening half-angle $b=\pi / 6$, and the $a$-rounded abs-val function is defined by $s_{a}(x):=(c / \sqrt{\pi}) e^{-x^{2} / a^{2}}+\operatorname{erf}(x / a) . \tau=0.05 . N=100$.

(a)

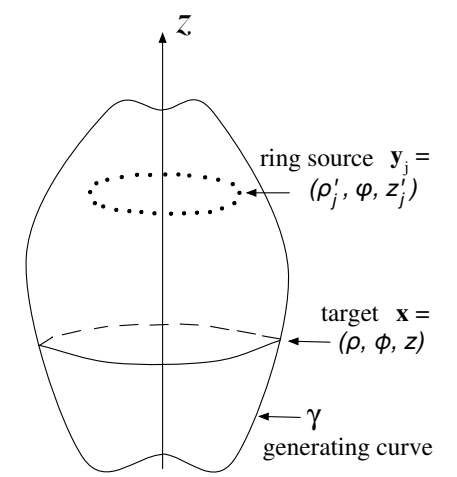

(b)

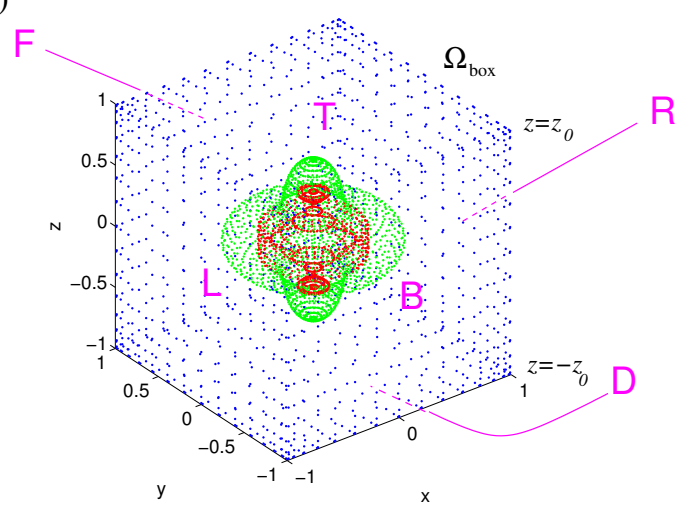

Figure 3: (a) Illustration of MFS for the exterior axisymmetric BVP showing a "ring charge" (dotted circle, where dots show approximation by individual point sources), and target ring (solid circle). (b) Geometry for periodization scheme: Surface points (green), MFS source points (red), and box wall discretization points (blue). 
Let the generating curve $\gamma$ for the body of revolution about the $z$-axis be parametrized by the smooth functions $(\rho(t), z(t))$ in the $\rho$ - $z$ plane, with $t \in[0, \pi]$. The "speed function" $s(t)=\sqrt{\left(\rho^{\prime}(t)\right)^{2}+\left(z^{\prime}(t)\right)^{2}}$ is non-vanishing. Note that the surface must also be smooth at $t=0$ and $t=\pi$, ie $z^{\prime}(0)=z^{\prime}(\pi)=0$. For example, the generating curves we test are shown in the lower part of Fig. 2; note that (c) is not formally smooth at $t=0$ and $\pi$, but is smooth to within double-precision rounding error because of the exponentially small deviation of the erf (error function) from \pm 1 at large arguments.

The right-hand side surface data is (10), $f=-\partial u^{\mathrm{i}} / \partial n$, which we approximate by the $P$-term truncated Fourier series

$$
f(\rho, z, \phi) \approx \sum_{n=-P / 2+1}^{P / 2} \hat{f}_{n}(\rho, z) e^{i n \phi}, \quad(\rho, z) \in \gamma
$$

with coefficients

$$
\hat{f}_{n}(\rho, z)=\frac{1}{2 \pi} \int_{0}^{2 \pi} f(\rho, z, \phi) e^{-i n \phi} d \phi, \quad n \in \mathbb{Z},
$$

whose accurate numerical evaluation we present shortly. $P$ is even, and the asymmetry of the $\pm P / 2$ terms will have no significant effect. The MFS source points $\left\{\left(\rho_{j}^{\prime}, z_{j}^{\prime}\right)\right\}_{j=1}^{N}$ live in the $\rho$ - $z$ plane, inside the curve $\gamma$; their location choice is discussed in the next section. The rotation of the $j$ th source point an angle $\varphi$ about the $z$-axis is denoted by

$$
\mathbf{y}_{j}(\varphi):=\left(\rho_{j}^{\prime}, \varphi, z_{j}^{\prime}\right)
$$

in cylindrical coordinates. Our representation for the scattered potential will be in terms of Fourier mode Helmholtz "ring sources", defining for the $n$th mode evaluated at target $\mathbf{x}$,

$$
\Phi_{n j}(\mathbf{x}):=\frac{1}{2 \pi} \int_{0}^{2 \pi} G_{k}\left(\mathbf{x}, \mathbf{y}_{j}(\varphi)\right) e^{-i n \varphi} d \varphi,
$$

recalling that $G_{k}$ is the free-space fundamental solution (1).

Our ansatz for the scattered potential is then

$$
u(\mathbf{x}) \approx \sum_{j=1}^{N} \sum_{n=-P / 2+1}^{P / 2} c_{n j} \Phi_{n j}(\mathbf{x})
$$

where the complex unknowns $c_{n j}$ are stacked into vectors $\mathbf{c}_{n}:=\left\{c_{n j}\right\}_{j=1}^{N}$ grouped by Fourier mode. We write $\eta:=$ $\left[\mathbf{c}_{-P / 2+1} ; \ldots ; \mathbf{c}_{P / 2}\right]$ for the $N P$-component column vector of all unknowns.

Imposing the boundary condition (10) means, for all surface points $\mathbf{x}=(\rho, \phi, z)$ in cylindrical coordinates,

$$
\sum_{n=-P / 2+1}^{P / 2} \sum_{j=1}^{N} c_{n j} \frac{1}{2 \pi} \int_{0}^{2 \pi} \frac{\partial G_{k}}{\partial n_{\mathbf{x}}}\left((\rho, \phi, z), \mathbf{y}_{j}(\varphi)\right) e^{-i n \varphi} d \varphi \approx \sum_{n=-P / 2+1}^{P / 2} \hat{f}_{n}(\rho, z) e^{i n \phi}, \quad(\rho, z) \in \gamma, \quad 0 \leq \phi<2 \pi
$$

By changing variable $\varphi$ to $\varphi-\phi$, and using orthogonality of the modes $e^{i n \phi}$ it is easy to derive that, for each mode $n$ separately,

$$
\sum_{j=1}^{N} c_{n j} A_{n}^{\prime}\left(\rho, z ; \rho_{j}^{\prime}, z_{j}^{\prime}\right) \approx \hat{f}_{n}(\rho, z), \quad(\rho, z) \in \gamma
$$

should hold, where the target normal-derivative of the $n$th "ring kernel" from source point $\left(\rho^{\prime}, z^{\prime}\right)$ to target $(\rho, z)$ (both lying in the $\rho-z$ plane) is

$$
A_{n}^{\prime}\left(\rho, z ; \rho^{\prime}, z^{\prime}\right):=\frac{1}{2 \pi} \int_{0}^{2 \pi} \frac{\partial G_{k}}{\partial n_{\mathbf{x}}}\left((\rho, 0, z),\left(\rho^{\prime}, \varphi, z^{\prime}\right)\right) e^{-i n \varphi} d \varphi
$$

where $n_{\mathbf{x}}$ is the normal to $\gamma$ at $(\rho, z)$. Finally, we enforce (17) at a set of $M$ collocation points $\left\{\left(\rho_{m}, z_{m}\right)\right\}_{m=1}^{M}$ on $\gamma$ to give the set of $P$ independent rectangular linear systems

$$
\sum_{j=1}^{N} A_{n}^{\prime}\left(\rho_{m}, z_{m} ; \rho_{j}^{\prime}, z_{j}^{\prime}\right) c_{n j}=\hat{f}_{n}\left(\rho_{m}, z_{m}\right), \quad m=1, \ldots, M, \quad n=-P / 2+1, \ldots, P / 2 .
$$


(a)

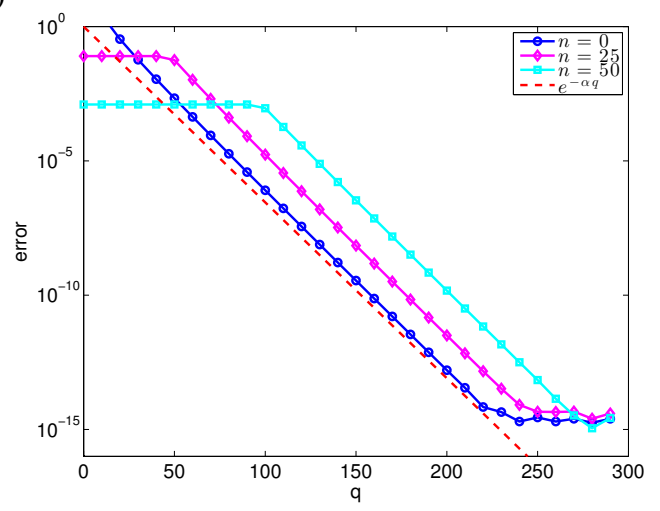

(b)

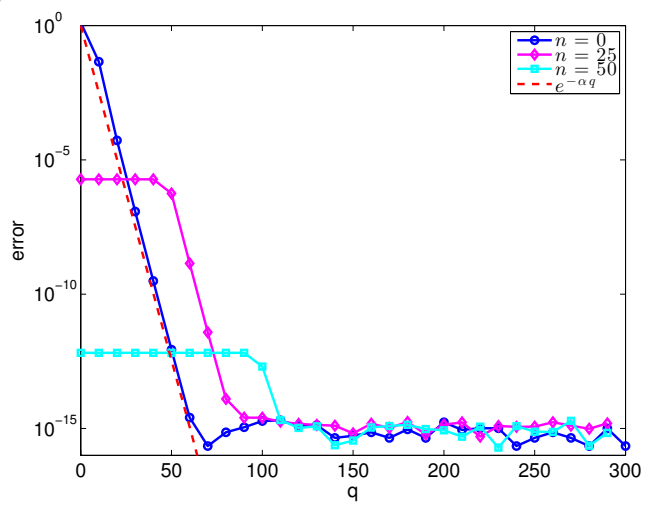

Figure 4: Convergence of the absolute error in evaluating the Helmholtz ring kernel (22), for the various $n$ shown in the caption with $k=10$. The shape we used is the one shown in Fig. 2(a) with $N=200, \tau=0.1$. The $q$-node periodic trapezoid rule is used. (a) Small distance: target $(\rho, z)=(0.5,0.49)$ and source $\left(\rho^{\prime}, z^{\prime}\right)=(0.45,0.44)$. (b) Large distance: target $(\rho, z)=(0.6,0.2)$ and source $\left(\rho^{\prime}, z^{\prime}\right)=(0.45,0.44)$. In each case the red line shows exponential convergence with rate equal to the supremum of the $\alpha$ predicted by Thm. 2 .

Typically $M$ must be slightly larger than $N$ to ensure accurate enforcement of the boundary condition. As is standard with the MFS, these systems are highly ill-conditioned, but if the sources are well chosen, are numerically consistent and possess a small coefficient norm $\left\|\mathbf{c}_{n}\right\|$. Assuming this norm is $O(1)$, if a backward-stable least-squares solve is used, machine precision accuracy can be reached despite the ill-conditioning [7]. For each $n$ independently the system is now solved in this way.

The set of linear systems may be written as

$$
\left[\begin{array}{ccc}
A_{-\frac{P}{2}+1}^{\prime} & & \\
& \ddots & \\
& & A_{\frac{P}{2}}^{\prime}
\end{array}\right]\left[\begin{array}{c}
\mathbf{c}_{-\frac{P}{2}+1} \\
\vdots \\
\mathbf{c}_{\frac{P}{2}}
\end{array}\right]=\left[\begin{array}{c}
\hat{f}_{-\frac{P}{2}+1} \\
\vdots \\
\hat{f}_{\frac{P}{2}}
\end{array}\right], \quad \text { summarized by } \quad A^{\prime} \eta=\hat{f},
$$

where $A^{\prime}$ has diagonal block structure, each of the $P$ diagonal blocks being a dense ill-conditioned rectangular matrix of size $M$ by $N$. Each of the $P$ systems will be solved independently in the least-squares sense using standard dense direct methods based on the QR decomposition (we use MATLAB's mldivide). The only remaining task is to fill their matrix elements and right-hand side vectors.

Numerical evaluation of the RHS $\hat{f}_{n}\left(\rho_{m}, z_{m}\right)$ for each curve point $m$ is done by applying the $q$-node periodic trapezoid rule quadrature [54, Sec. 12.1] to (14), i.e.,

$$
\hat{f}_{n}(\rho, z) \approx \frac{1}{q} \sum_{l=1}^{q} f(\rho, 2 \pi l / q, z) e^{-2 \pi i n l / q}=\left(\mathcal{F}^{-1} \mathbf{f}\right)_{n}, \quad \text { where } \mathbf{f}:=\{f(\rho, 2 \pi l / q, z)\}_{l=1}^{q}, \quad n \in \mathbb{Z},
$$

where $\mathcal{F}$ is the $q$-point discrete Fourier transform matrix. The error in this quadrature formula ("aliasing" error) can be bounded by the sum of the magnitudes of the exact Fourier coefficients $\hat{f}_{n}$ for which $|n| \geq q / 2$ [72, (3.10)]. Since, for an accurate BVP solution, $P$ must already be large enough that coefficients are small beyond $P / 2$, we may choose $q=P$ without losing accuracy due to quadrature in (21). Note that (21) can be evaluated for all $-P / 2<n \leq P / 2$ at once using a single FFT in $O(P \log P)$ time. Since $u^{\mathrm{i}}$ is analytic and the surface smooth, the Fourier data $\hat{f}_{n}$ decays super-algebraically with $|n|$, and thus we expect similar convergence with respect to $P$.

We use a similar idea to evaluate matrix elements of $A_{n}^{\prime}$, which we now describe.

\subsection{Evaluation of the axisymmetric Helmholtz ring kernel}

The $m, j$ matrix element of $A_{n}^{\prime}$ in the linear system (19) is given by the target normal-derivative of the Helmholtz ring kernel

$$
A_{n}^{\prime}\left(\rho_{m}, z_{m} ; \rho_{j}^{\prime}, z_{j}^{\prime}\right)=\int_{0}^{2 \pi} \frac{\partial G_{k}}{\partial n_{m}}\left(\left(\rho_{m}, 0, z_{m}\right),\left(\rho_{j}^{\prime}, \varphi, z_{j}^{\prime}\right)\right) e^{-i n \varphi} d \varphi
$$


where $n_{m}$ indicates the normal to $\gamma$ at the point $\left(\rho_{m}, z_{m}\right)$. For the transmission BVP (Sec. 3.4) we will also need the ring kernel value matrix element

$$
A_{n}\left(\rho_{m}, z_{m} ; \rho_{j}^{\prime}, z_{j}^{\prime}\right):=\int_{0}^{2 \pi} G_{k}\left(\left(\rho_{m}, 0, z_{m}\right),\left(\rho_{j}^{\prime}, \varphi, z_{j}^{\prime}\right)\right) e^{-i n \varphi} d \varphi
$$

Analytic series for this kernel are presented by Conway-Cohl [24], and evaluation methods for them in the BIE setting, where the target may come very close to the source, are given in [74, 41, 42, 43]. However, in the MFS setting, the target $(\rho, z)$ is separated from the source $\left(\rho^{\prime}, z^{\prime}\right)$ by a distance of at least a few times the quadrature node spacing (see Sec. 3.2), and hence the periodic trapezoid rule quadrature

$$
A_{n}\left(\rho_{m}, z_{m} ; \rho_{j}^{\prime}, z_{j}^{\prime}\right) \approx \frac{1}{q} \sum_{l=1}^{q} G_{k}\left(\left(\rho_{m}, 0, z_{m}\right),\left(\rho_{j}^{\prime}, 2 \pi l / q, z_{j}^{\prime}\right)\right) e^{-2 \pi i n l / q}
$$

will be efficient for evaluating (22). In fact, we will have exponential convergence with a rate controlled by the source-target separation in the $\rho-z$ plane, as the following theorem shows.

Theorem 2. The q-node periodic trapezoid rule applied to the Helmholtz ring kernel or it's target normal derivative is exponentially convergent with known rate $\alpha$. Namely, for any $\alpha<\operatorname{Im} \cos ^{-1}\left(\frac{\left(\rho-\rho^{\prime}\right)^{2}+\left(z-z^{\prime}\right)^{2}}{2 \rho \rho^{\prime}}+1\right)$, there is a constant $C$ independent of $q$ such that

$$
\left|\frac{1}{2 \pi} \int_{0}^{2 \pi} G_{k}\left((\rho, 0, z),\left(\rho^{\prime}, \varphi, z^{\prime}\right)\right) e^{-i n \varphi} d \varphi-\frac{1}{q} \sum_{l=1}^{q} G_{k}\left((\rho, 0, z),\left(\rho^{\prime}, 2 \pi l / q, z^{\prime}\right)\right) e^{-2 \pi i n l / q}\right| \leq C e^{-\alpha q}
$$

for all sufficiently large $q$, and the same holds when $G_{k}$ is replaced by $\frac{\partial G_{k}}{\partial n_{\mathbf{x}}}, n_{\mathbf{x}}$ being the normal to $\gamma$ at $(\rho, z)$.

Proof. The theorem of Davis [26] states that the periodic trapezoid rule is exponentially convergent for a periodic analytic integrand. Specifically, for the periodic interval $\varphi \in[0,2 \pi)$, if an integrand $f(\varphi)$ can be analytically continued off the real axis to a bounded $2 \pi$-periodic analytic function in the strip $|\operatorname{Im} \varphi| \leq \alpha$, then

$$
\left|\frac{1}{2 \pi} \int_{0}^{2 \pi} f(\varphi) d \varphi-\frac{1}{q} \sum_{l=1}^{q} f(2 \pi l / q)\right| \leq C e^{-\alpha q}
$$

holds for some constant $C$ and all sufficiently large $q$. It only remains to understand the region of analyticity of the integrands

$$
f(\varphi):=G_{k}(\mathbf{x}, \mathbf{y}) e^{-i n \varphi} \quad \text { and } \quad f(\varphi):=\frac{\partial G_{k}}{\partial n_{\mathbf{x}}}(\mathbf{x}, \mathbf{y}) e^{-i n \varphi}, \quad \text { where } \mathbf{x}=(\rho, 0, z), \quad \mathbf{y}=\left(\rho^{\prime}, \varphi, z^{\prime}\right),
$$

with respect to the variable $\varphi$. The Green's function (1) is analytic unless the distance between source and target vanishes, ie

$$
0=|\mathbf{x}-\mathbf{y}|=\sqrt{\left(\rho-\rho^{\prime} \cos \varphi\right)^{2}+\left(\rho^{\prime} \sin \varphi\right)^{2}+\left(z-z^{\prime}\right)^{2}} .
$$

Solving for $\varphi$ gives locations of the singularities in the complex $\varphi$ plane,

$$
\varphi=\cos ^{-1}\left(\frac{\left(\rho-\rho^{\prime}\right)^{2}+\left(z-z^{\prime}\right)^{2}}{2 \rho \rho^{\prime}}+1\right) .
$$

These occur with $2 \pi$ periodicity in the real direction, in pairs symmetric about the real axis. Thus the two integrands $f(\varphi)$ are analytic and bounded in any strip $|\operatorname{Im} \varphi| \leq \alpha$ for $\alpha$ given in the statement of the theorem. Applying the Davis theorem completes the proof. 
Note that in the limit of small separation, by expanding the cosine, the maximum convergence rate guaranteed by Thm. 2 is

$$
\alpha \approx \frac{\sqrt{\left(\rho-\rho^{\prime}\right)^{2}+\left(z-z^{\prime}\right)^{2}}}{\rho},
$$

that is, equal to ratio of the source-target separation in the $\rho-z$ plane to $\rho$.

In Fig. 4 we demonstrate Thm. 2 numerically in a typical axisymmetric MFS setting. Panel (a) shows the error convergence in evaluating (22), i.e. one matrix element of $A_{n}$, for a small source-target separation of 0.071 . The convergence rate is slow, independent of $n$, and observed to match very closely the upper bound on the rate $\alpha$ predicted by the theorem. Panel (b) shows a much larger distance, and much faster convergence, again with rate independent of $n$ and as predicted by the theorem. The results for the normal derivative ring kernel (18) are similar.

Notice that the value of $q$ after which converge starts depends on the Fourier mode $n$, and that, as $n$ increases this adds roughly $|n|$ to the $q$ value at which convergence to machine precision is reached. We explain this as follows. In the Davis theorem the constant $C$ asymptotic to $4 \pi \sup _{\operatorname{Im} \varphi= \pm \alpha}|f(\varphi)|$, i.e. a bound on the size of $f$ in the strip [54, Thm. 12.6]. The factor $e^{-i n \varphi}$ in the integrand contributes a maximum value within the strip of $e^{\alpha|n|}$, which is the dominates $C$. Thus around $|n|$ extra quadrature nodes are needed to reach the error level for $n=0$.

Remark 2. Since the individual terms in the quadrature sum (23) are of size O(1) for a typical source-target separations in a unit-sized obstacle, we can get convergence to machine precision in the absolute error, but not the relative error, of each matrix element of $A_{n}^{\prime}$ or $A_{n}$. However, absolute error is the relevant quantity controlling final accuracy since the solution field is summed over Fourier modes (16).

In the rest of this paper, we fix $q$ to the same value for filling all matrix elements in $A_{n}^{\prime}$ or $A_{n}$, determined by a convergence test as shown in Sec. 3.3. It would be possible to use that above theorem to choose $q$ differently to be more optimal for each matrix element (source-target distance); however, since the matrix filling is not a large part of the total solution time there would not be much benefit. For evaluation of the solution potential (16) on other periodic images of the obstacle, we find that a fixed value $q=P$ is adequate.

\subsection{Choice of source point locations}

The performance of the MFS depends critically on the choice of the curve $\Gamma_{-}$on which the $N$ sources lie [31,28]. For analytic boundaries there are strong theoretical results. In this case, Katsurada [51] proved that, in exact arithmetic, the MFS has exponential convergence for the 2D Dirichlet Laplace $(k=0)$ interior BVP, with $M=N$ and points chosen equally spaced in the parametrization of the curves $\Gamma$ and $\Gamma_{-}$. This was generalized to all analytic kernels (thus including the Helmholtz kernel) by Kangro [48], and recently to the 3D Maxwell case when the source points $\left\{\mathbf{y}_{j}\right\}$ are the nodes of an exponentially convergent quadrature on $\Gamma_{-}$[49] (presumably a similar proof would apply for Helmholtz).

However, in floating-point arithmetic another constraint arises, associated with the exponentially large condition number of the MFS system matrix. Namely, the coefficient norm $\|\mathbf{c}\|$ should remain small, i.e. $O(1)$. It is conjectured that this happens if and only if the exterior solution $u$ can be continued as a regular solution to the PDE inside $\Omega$ up to and including $\Gamma_{-}$, in other words if $\Gamma_{-}$encloses all of the singularities in the continuation of $u$ [7, Conj. 12]. This is suggested by theory on the continuous first-kind integral equation [56, p. 1238] [28, Thm. 2.4], and numerical results where the singularities are known (via the Schwartz function) [7]. If $\Gamma_{-}$is placed too far from the surface, it will not enclose these singularities, and exponential blowup of $\|\mathbf{c}\|$ results, causing rounding error which limits the solution error for $u$.

Now we compare the $N$-convergence of the error in our setting, for various different methods for choosing source locations. We use the notation that the sources $\mathbf{y}_{j}:=\left(\rho_{j}, z_{j}\right), j=1, \ldots, N$ lying on a curve $\Gamma_{-}$are displaced from $N$ surface points $\mathbf{x}_{j}:=\left(\rho\left(t_{j}\right), z\left(t_{j}\right)\right)$ with parameters $t_{j}=\pi(j-1 / 2) / N, j=1 \ldots, N$, uniformly spaced on the generating curve $\gamma$. The methods we compare are:

(a) displacement by a constant distance $\tau$ in the normal direction,

(b) displacement by a distance $\tau s\left(t_{j}\right)$ in the normal direction, where $s(t)$ is the speed function, and

(c) displacement in the "imaginary direction" by complexification of the boundary parametrization, i.e. $\mathbf{y}_{j}:=\left(\rho\left(t_{j}+\right.\right.$ $\left.i \tau), z\left(t_{j}+i \tau\right)\right)$. This is a simplification of methods from [7]. 
(a)

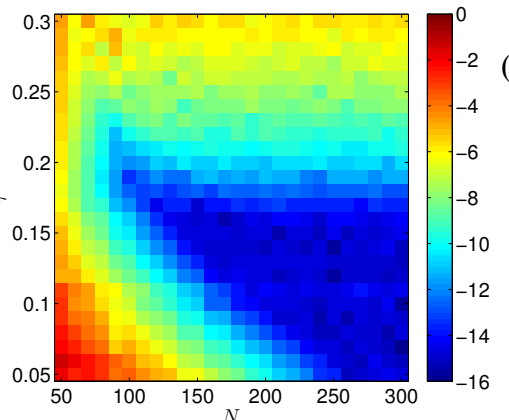

(b)

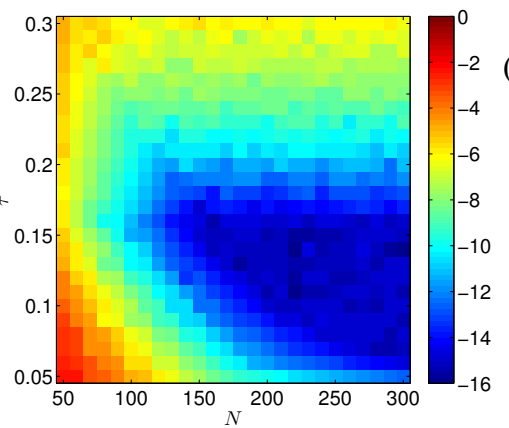

(c)

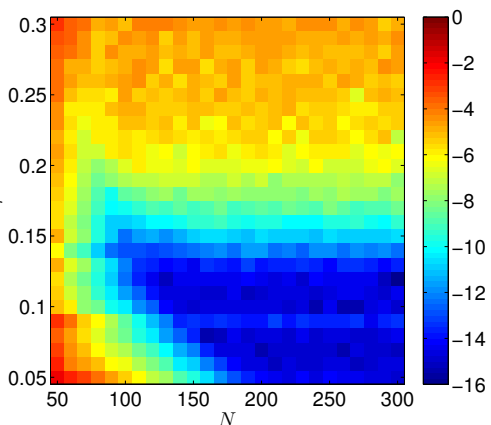

d)

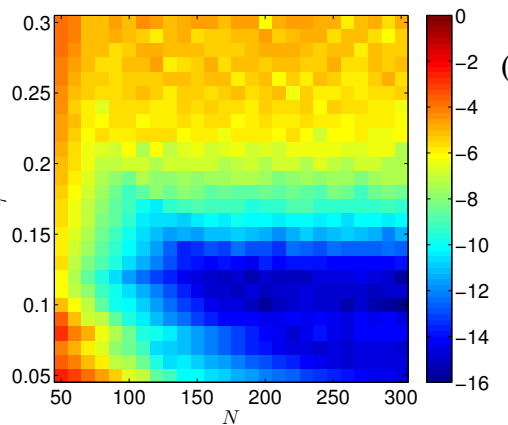

(e)

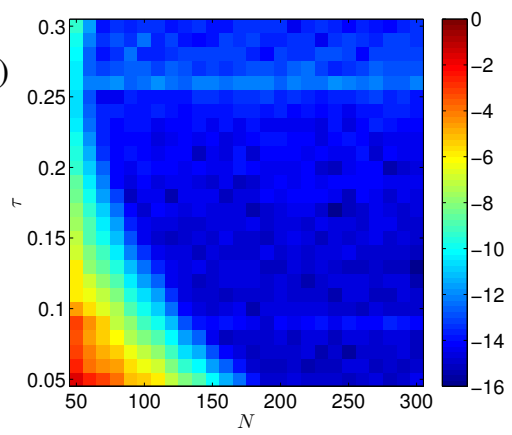

(f)

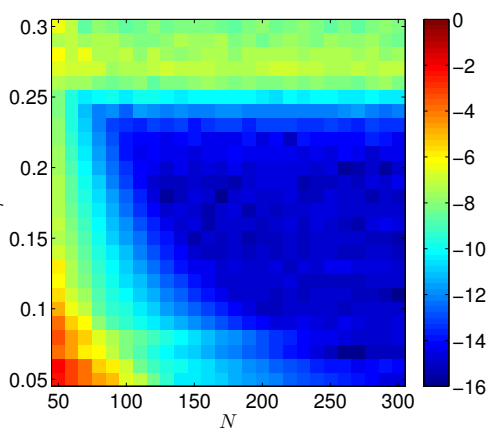

Figure 5: Convergence of error at a single distant point in the solution of Neumann Helmholtz scattering problems at $k=10$ via the MFS for various source point displacement schemes, for the boundary curve the "smooth" shape of Fig. $2(\mathrm{a}), f(\theta)=1+0.3 \cos 4 \theta$ for $\theta \in[0, \pi]$. The top row of plots shows the error for solution of a 2D BVP using a boundary curve reflected to form a closed curve $\theta \in[0,2 \pi]$; the bottom row shows error for the corresponding 3D axisymmetric BVP. $N$ is the number of source points in $\theta \in[0, \pi]$. (a) and (b): constant displacement from surface points along the normal vector. (c) and (d): scaling the displacement in proportion to the "speed" $|s(t)|$. (e) and (f): constant displacement in the imaginary parameter direction. In each plot "aliasing" error due to sources too close to the boundary dominates in the bottom left, and growth of error in the vertical direction is caused by round-off due to growing coefficient norms $\|\mathbf{c}\|$. 
Note that all methods depend upon the parametrization, and we assume that one is used for which $\left\{\mathbf{x}_{j}\right\}$ provides a good quadrature scheme on $\gamma$ (combined with its reflection).

In Fig. 5 we compare these three methods for solving the Neumann Helmholtz scattering problem in the smooth shape shown in Fig. 2(a), using a dense direct linear In fact we compare the 2D case of plane-wave scattering from the curve (closed by combining with its reflection about the $z$ axis), against the results for the 3D axisymmetric case, using a dense direct solve for each Fourier mode. Two conclusions stand out: 1) the 2D BVP is an excellent indicator of error performance in the 3D axisymmetric case, and 2) the complexification method is at least as good as the other methods at small $\tau$, and is more forgiving at large $\tau$. Thus, in the rest of this work we choose the complexification method.

The tested wavenumber $k=10$ is not high; we find that at $k=30$ the blow-up at large $\tau$ is more severe even for the complexification method. Thus we choose $\tau$ empirically so that it gives rapid $N$ convergence but does not blow up at high $k$. This we assess via experimentation with the 2D BVP, since is very rapid to run (taking a fraction of a second per solve), and is demonstrated to reflect the 3D performance. In future work we will present an automated method for choosing $\tau$ and $N$.

Remark 3. The monopole ("single-layer") MFS representation that we use fails to be a complete representation of exterior radiative potentials if the surface on which the sources lie has an interior Neumann eigenvalue equal to $k^{2}$ [28, Sec. 2.1]. In practice, this can be detected by a poor boundary error ( $\epsilon_{1}$ in Sec. 3.3 below), and $\tau$ changed slightly. In our experiments we have never had to make such a change.

\subsection{Isolated obstacle Neumann scattering convergence tests}

Thus far we have made three approximations, each of which involves a parameter: 1) the MFS approximation involving $N$ source points, 2) the Fourier series truncation to $P$ terms, and 3) the quadrature evaluation of the ring kernel via $q$ periodic trapezoid nodes. We now show convergence results for these parameters in the context of solving the one-obstacle scattering problem (8), (10) and (12). The first row of Fig. 6 shows the convergence with respect to $N$, the second row with respect to $P$, and the last row with respect to $q$. In each case the parameters not under convergence study are fixed at their converged values. Two types of error are shown:

- $\epsilon_{1}$ : Absolute $L^{2}$ error in the boundary condition (10), estimated at a grid of $128 \times 128$ points on $\partial \Omega$ very few of which coincide with collocation points $\mathbf{x}_{i}$, and

- $\epsilon_{2}$ : relative error in the value of the scattered potential $u$ at a distant point near to $(10,10,10)$, compared to its converged value.

Fig. 6 is consistent with exponential convergence in all three parameters, with the values where convergence starts being larger for large wavenumber $k$. The convergence of the two error measures is similar, usually differing by a fixed constant, and saturating at different values. Even in the case of a resonant object at high frequency (the plots in the right column), 9 digits are reached in the boundary condition error, and 12 digits in the solution $u$ at a distant point.

\subsection{The transmission case}

Now we briefly describe how the above axisymmetric MFS scheme is adjusted for the transmission case (11a)(11c). As before, the scattered wave $u$ is represented by the ring kernel MFS source sum (16) with source locations $\left(\rho_{j}^{\prime}, z_{j}^{\prime}\right)$ inside the obstacle. In addition the scattered wave $u^{-}$inside the obstacle is represented by a similar sum

$$
u^{-} \approx \sum_{j=1}^{N} \sum_{n=-P / 2+1}^{P / 2} c_{n j}^{-} \Phi_{n j}^{-}, \quad \text { where } \quad \Phi_{n j}^{-}(\mathbf{x}):=\frac{1}{2 \pi} \int_{0}^{2 \pi} G_{k_{-}}\left(\mathbf{x}, \mathbf{y}_{j}^{-}(\varphi)\right) e^{-i n \varphi} d \varphi, \quad \mathbf{y}_{j}^{-}(\varphi):=\left(\rho_{j}^{\prime \prime}, \varphi, z_{j}^{\prime \prime}\right),
$$

with sources $\left(\rho_{j}^{\prime \prime}, z_{j}^{\prime \prime}\right)$ lying outside the obstacle. The set-up is as in Fig. 1(c) (which shows a 2D sketch), an example being the green points in Fig. 2(b). These exterior source locations are chosen by negating $\tau$ in the source point 
(a)

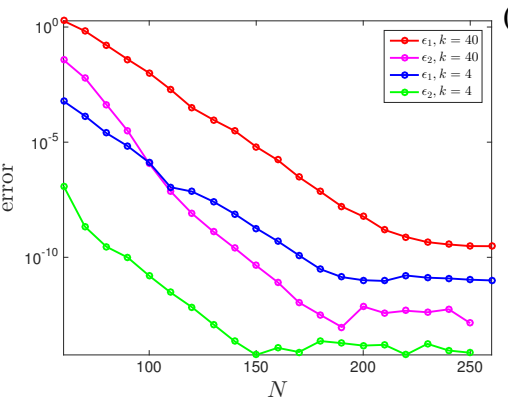

(b)

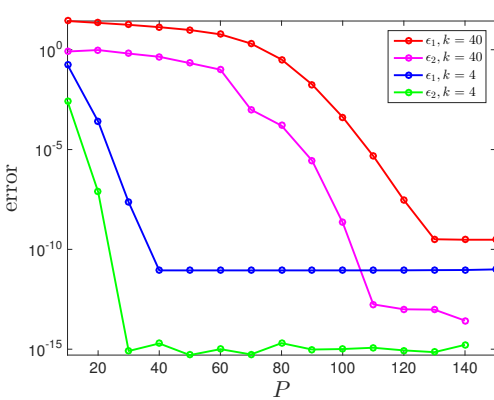

(c)

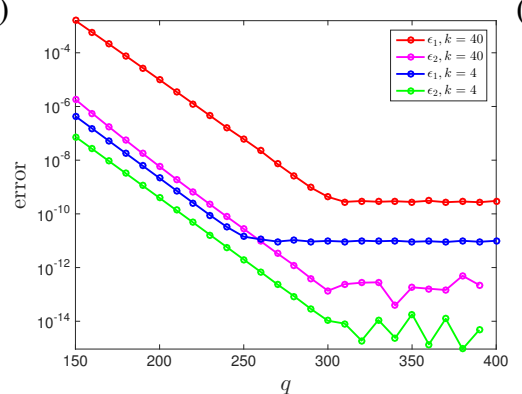

(d)

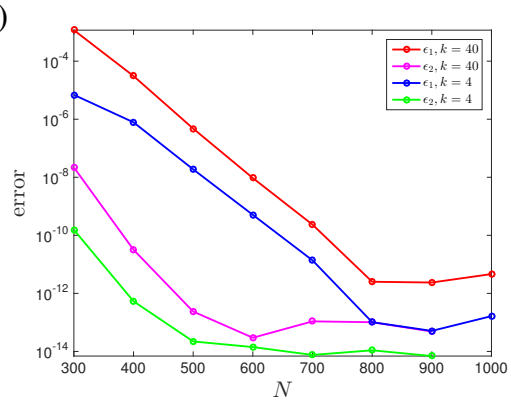

(e)

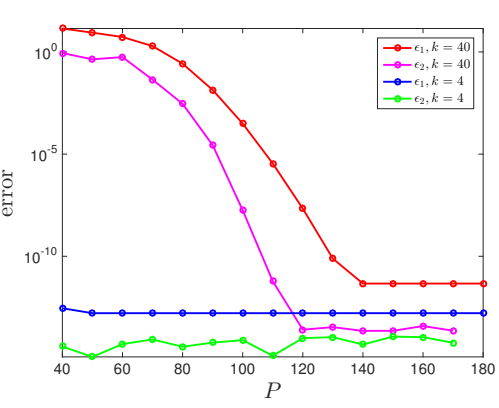

(f)

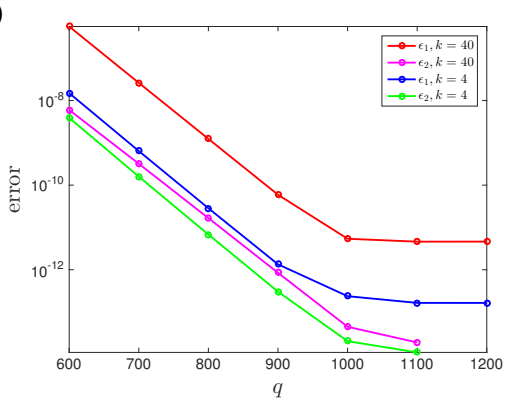

(g)

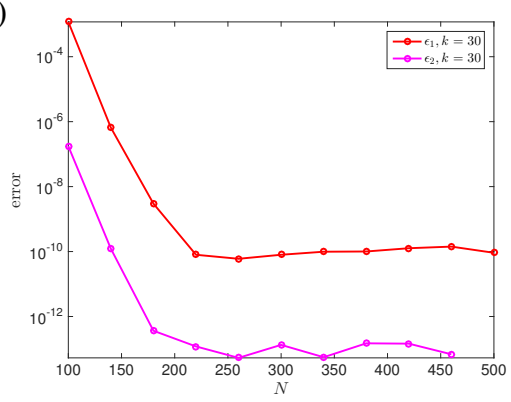

(h)

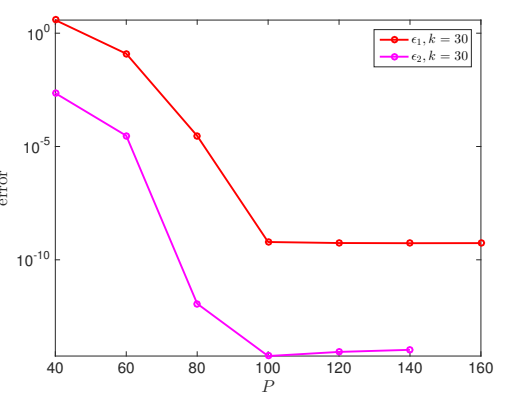

(i)

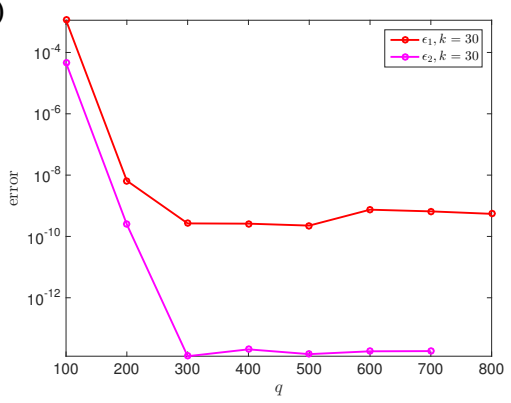

Figure 6: Error convergence for the 3D axisymmetric MFS solution of the Helmholtz Neumann scattering BVP for low and high frequencies (wavenumber $k$ is shown in legends). $\epsilon_{1}$ estimates the boundary condition error, $\epsilon_{2}$ the solution error at a distant point. Left column: "smooth" shape from Fig. 2(a), with $\tau=0.1$. (a) varying $N$, (b) varying $P$, (c) varying $q$. The fixed (converges) parameter values are $N=260, P=150$, $q=400$. Middle column: "wiggly" shape from Fig. 2(b), with $\tau=0.03$. Fixed values are $N=1000, P=180, q=1200$. Right column: cup shape from Fig. 2(c), with $\tau=0.1$. Fixed values are $N=500, P=160, q=800$. In all cases $M \approx 1.2 N$. 
location algorithms in Sec. 3.2. Imposing the value and normal derivative matching conditions (11b) and (11c) gives the linear system

$$
\left[\begin{array}{cc}
A & -A^{-} \\
A^{\prime} & -A^{\prime-}
\end{array}\right]\left[\begin{array}{c}
\eta \\
\eta^{-}
\end{array}\right]=\left[\begin{array}{c}
\hat{f} \\
\hat{f}^{\prime}
\end{array}\right]
$$

where each of the four blocks is block diagonal, with rectangular diagonal blocks $A_{n}, A_{n}^{-}, A_{n}^{\prime}, A_{n}^{\prime-}$ respectively, $-P / 2<$ $n \leq P / 2$. The new blocks $A_{n}^{-}$and $A_{n}^{\prime-}$ are identical to (22) and (18) respectively, but with $k$ replaced by $k_{-}, \rho_{j}^{\prime}$ by $\rho_{j}^{\prime \prime}$, and $z_{j}^{\prime}$ by $z_{j}^{\prime \prime}$. The RHS data blocks $\hat{f_{n}}$ and $\hat{f}_{n}^{\prime}$ are constructed as before via (21) using $f=-u^{\mathrm{i}}$ and $f^{\prime}=-\frac{\partial u^{\mathrm{i}}}{\partial n}$ respectively. Abusing notation somewhat, we will summarize both (20) and (25) by the linear system

$$
A_{0} \eta=\hat{f} .
$$

where $A_{0}$ is of size $M P$-by-NP in the Neumann case, and size $2 M P$-by-2NP in the transmission case.

\section{Periodizing scheme}

We choose a cuboid unit cell $\Omega_{\mathrm{box}}$ of sizes $e_{x}$ and $e_{y}$ in the $x$ and $y$ axes respectively, and truncated in the vertical direction to $z \in\left[-z_{0}, z_{0}\right]$ such as to contain $\Omega$. The rectangular walls enclosing $\Omega_{\text {box }}$ are left $L$, right $R=L+\left(e_{x}, 0,0\right)$, back $B$, front $F=B+\left(0, e_{y}, 0\right)$, and downwards $D$ at $z=-z_{0}$ and top $T$ at $z=z_{0}$; see Fig. 3(b). All normals point in the positive coordinate directions. We use the abbreviation $u_{L}$ to mean $u$ restricted to $L$, and $u_{n L}$ to mean $\partial u / \partial n$ restricted to $L$. We now reformulate the periodic BVP on $\Omega_{\text {box }}$ alone, as in [13,21]. Taking for simplicity the Neumann case, $u$ satisfies (8) in $\Omega_{\mathrm{box}},(10)$, and we match Cauchy data on the four side walls giving

$$
\begin{aligned}
u_{R}-\alpha u_{L} & =0 \\
u_{n R}-\alpha u_{n L} & =0 \\
u_{F}-\beta u_{B} & =0 \\
u_{n F}-\beta u_{n B} & =0,
\end{aligned}
$$

which (because of the unique continuation property of an elliptic PDE) is equivalent to quasi-periodicity (6), and finally match Cauchy data to Rayleigh-Bloch expansions (9a)-(9b) on the top and bottom walls,

$$
\begin{aligned}
u\left(x, y, z_{0}\right) & =\sum_{m, n \in \mathbf{Z}} a_{m n} \exp i\left[\kappa_{x}^{m} x+\kappa_{y}^{n} y\right], \quad\left(x, y, z_{0}\right) \in T \\
u_{z}\left(x, y, z_{0}\right) & =\sum_{m, n \in \mathbf{Z}} i \kappa_{z}^{(m, n)} a_{m n} \exp i\left[\kappa_{x}^{m} x+\kappa_{y}^{n} y\right], \quad\left(x, y, z_{0}\right) \in T \\
u\left(x, y,-z_{0}\right) & =\sum_{m, n \in \mathbf{Z}} b_{m n} \exp i\left[\kappa_{x}^{m} x+\kappa_{y}^{n} y\right], \quad\left(x, y,-z_{0}\right) \in D \\
u_{z}\left(x, y,-z_{0}\right) & =-\sum_{m, n \in \mathbf{Z}} i \kappa_{z}^{(m, n)} b_{m n} \exp i\left[\kappa_{x}^{m} x+\kappa_{y}^{n} y\right], \quad\left(x, y,-z_{0}\right) \in D .
\end{aligned}
$$

The solution $u$ in $\Omega_{\mathrm{box}}$ is represented as

$$
u(\mathbf{x}) \approx \sum_{j=1}^{N} \sum_{n=-P / 2+1}^{P / 2} c_{n j} \Phi_{n j}^{\mathrm{near}}(\mathbf{x})+\sum_{l=0}^{p} \sum_{m=-l}^{l} d_{l m} j_{l}(k r) Y_{l m}(\theta, \phi)
$$

where the sum of the ring kernel over the nearest neighbors in the lattice is

$$
\Phi_{n j}^{\text {near }}(\mathbf{x}):=\sum_{|m|,|n| \leq 1} \alpha^{m} \beta^{n} \frac{1}{2 \pi} \int_{0}^{2 \pi} G_{k}\left(\mathbf{x}, \mathbf{y}_{j}(\varphi)+m \mathbf{e}_{x}+n \mathbf{e}_{y}\right) e^{-i n \varphi} d \varphi .
$$

The unknowns are $\eta:=\left\{c_{n j}\right\}_{j=1, \ldots, N,-P / 2<n \leq P / 2}, \mathbf{d}:=\left\{d_{l m}\right\}_{l=0, \ldots, p,|m| \leq l}$, and we restrict the Rayleigh-Bloch expansions (31)-(34) to the $x y$-plane wavevectors of magnitude at most $\pi N_{0}$, where $N_{0}$ is a convergence parameter, so a := $\left\{a_{m n}\right\}_{\left(\kappa_{x}^{m}\right)^{2}+\left(\kappa_{y}^{n}\right)^{2} \leq \pi^{2} N_{0}^{2}}$ and $\mathbf{b}:=\left\{b_{m n}\right\}_{\left(\kappa_{x}^{m}\right)^{2}+\left(\kappa_{y}^{n}\right)^{2} \leq \pi^{2} N_{0}^{2}}$. 
Remark 4 (Choice of $z_{0}$ ). If the vertical extent of $\Omega$ is not larger than the period, then choosing $\Omega_{b o x}$ roughly cubical is efficient. Generally, $z_{0}$ must be at least some distance (say, $1 / 4$ period) above the extent of $\Omega$ in order that the Rayleigh-Bloch expansions converge rapidly; on the other hand if $z_{0}$ is too large, the spherical harmonic convergence rate on the faces is reduced. For high aspect ratio obstacles (not tested in this work), either more local terms are needed in (36), or an elongated proxy surface could be used (in the style of App. A; also see [9]).

\subsection{Full linear system}

We now build the full linear system that the stacked column vector of all unknowns $[\eta ; \mathbf{d} ; \mathbf{a} ; \mathbf{b}]$ must satisfy, by enforcing the boundary conditions, but also the quasi-periodicity and upward and downward radiation conditions.

The first block row arises from enforcing (10) on the boundary nodes on $\partial \Omega$ as in Sec. 3, giving

$$
A \eta+\tilde{B} \mathbf{d}=\hat{f}
$$

where

$$
A=A_{0}+A_{\text {else }},
$$

$A_{0}$ being the direct self-interaction of the obstacle as in (26) (i.e. $A_{0}$ is zero apart from diagonal blocks $A_{n}^{-}$). Rather than writing a long formula for the matrix elements of $A_{\text {else }}$, it is more useful to describe its action: $A_{\text {else }} \eta$ is the set of Fourier series coefficients of the normal-derivatives of $u$ on the target rings $\left\{\rho_{m}, z_{m}\right\}_{m=1}^{M}$ due to the eight phased ring sources from the first term in (35), omitting the central copy $m=n=0$. In practice we may compute $A_{\text {else }} \eta$ efficiently as follows: the quadrature (23) is equivalent to replacing each ring kernel by $q$ point sources with strengths given by the FFT of the coefficients $c_{n j}$ in the $n$ direction. The FMM evaluates the potential on the $8 q N$ sources at a set of $q M$ trapezoidal-node targets on rings on $\partial \Omega$, and the FFT is finally used to convert back to Fourier coefficients as in (21). A quadrature parameter $q=P$ is sufficient here, since interactions in $A_{\text {else }}$ are distant. The cost of applying $A_{\text {else }}$ (assuming $M=O(N))$ is thus $O(N P \log P)$, although it is typically dominated by the $O(N P)$ of the FMM.

The matrix $\tilde{B}$ in (37) has elements that can approximated by the periodic trapezoid rule,

$$
\tilde{B}_{n i, l m}=\frac{1}{2 \pi} \int_{0}^{2 \pi} j_{l}\left(k r_{i}\right) Y_{l m}\left(\theta_{i}, \phi\right) e^{-i n \phi} d \phi \approx \frac{1}{q} \sum_{s=1}^{q} j_{l}\left(k r_{i}\right) Y_{l m}\left(\theta_{i}, 2 \pi s / q\right) e^{-2 \pi i n s / q},
$$

where $r_{i}=\sqrt{\rho_{i}^{2}+z_{i}^{2}}$ and $\theta_{i}=\tan ^{-1} z_{i} / \rho_{i}$ are the spherical coordinates of the $i$ th boundary point on $\gamma$. Note that if the axis of the obstacle is aligned with the $z$-axis of the spherical harmonic expansion, simplifications apply making $\tilde{B}$ sparse; for more generality we leave it in the above form. We fill the matrix $\tilde{B}$ once and for all at a given $k$, at a cost $O\left(N P p^{2}\right)$.

The remaining block rows of the full linear system result from substituting (35) into (27)-(34), or, more specifically, (27)-(34) evaluated at collocation nodes lying on the faces. For these nodes we use the nodes from a $M_{1}$-by- $M_{1}$ product Gaussian quadrature on each rectangular face, with $M_{1}$ chosen large enough that further changes have no effect on the solution. We pick $M_{1}$ to be $M_{1} \approx 4 \frac{k}{\pi}$ so that we can guarantee 4 points per wavelength in each direction of the rectangular faces.

The resulting full system has the form

$$
\left[\begin{array}{c|ccc}
A & \tilde{B} & 0 & 0 \\
\hline C_{L, R} & S_{L, R} & 0 & 0 \\
C_{n L, n R} & S_{n L, n R} & 0 & 0 \\
C_{B, F} & S_{B, F} & 0 & 0 \\
C_{n B, n F} & S_{n B, n F} & 0 & 0 \\
C_{T} & S_{T} & -W_{T} & 0 \\
C_{n T} & S_{n T} & -W_{n T} & 0 \\
C_{D} & S_{D} & 0 & -W_{D} \\
C_{n D} & S_{n D} & 0 & -W_{n D}
\end{array}\right]\left[\begin{array}{c}
\eta \\
\mathbf{d} \\
\mathbf{a} \\
\mathbf{b}
\end{array}\right]=\left[\begin{array}{c}
\hat{f} \\
0 \\
0 \\
0 \\
0 \\
0 \\
0 \\
0 \\
0
\end{array}\right], \quad \text { summarized as }\left[\begin{array}{cc}
A & B \\
C & Q
\end{array}\right]\left[\begin{array}{l}
\eta \\
\xi
\end{array}\right]=\left[\begin{array}{c}
\hat{f} \\
0
\end{array}\right],
$$

where $\xi:=[\mathbf{d} ; \mathbf{a} ; \mathbf{b}]$ groups the periodizing unknowns, and where the division of the matrix blocks defining the $2 \times 2$ block notation is shown by lines. 
The recipe for filling the above blocks is implicit in the above description, and rather than give their full formulae (see [21] for full formulae in a related 2D problem), we explain their meaning. The $A$ block is already given in (38). The $B$ block is merely $\tilde{B}$ padded with zeros to its right. The $C$ block describes the effect of the ring kernel coefficients $c_{n j}$ in $\eta$ on the discrepancies, namely the left-hand sides of (27)-(30), and on the Cauchy data on $T$ and $D$. Significant cancellation occurs in the upper four $C$ blocks, identical to that in [8]. Consider the $3 \times 3$ grid of nearest neighbors in phased image sums such as (36), in the block $C_{L, R}$ : the effect of the left-most six on the $L$ wall cancels the effect of the right-most six on the $R$ wall, leaving only 6 of the original 18 terms. The remaining terms correspond only to distant interactions: the effect of the right-most three on $L$ and the left-most three on $R$. Identical cancellations occur in the next three blocks of $C$. As discussed in [8], this also allows the scheme to work well even if $\Omega$ is not confined within $\Omega_{\text {box }}$ and wall-obstacle intersections occur. The $C$ matrix is filled densely once and for all at each $k$, at a cost $O\left(M_{1}^{2} N P\right)$.

$Q$ has $8 M_{1}^{2}$ rows and $N_{Q}:=(p+1)^{2}+O\left(N_{0}^{2}\right)$ columns; note that this is independent of $\mathcal{N}$, the number of obstacle unknowns. However, both dimensions of $Q$ grow with wavenumber as $O\left(k^{2}\right)$. We fill its non-zero blocks densely by evaluation of spherical harmonics (for the $S$ blocks) and plane-wave expansions (for the $W$ blocks). The $S$ blocks give the effect of the spherical harmonic basis on the discrepancies and Cauchy data on $T$ and $D$, while the $W$ blocks give the effect of the Rayleigh-Bloch expansions on and Cauchy data on $T$ and $D$. The negative signs in the latter account for the fact that the jumps in Cauchy data should vanish.

The transmission case is similar to the above, with $A$ twice the size in each dimension (with $A_{0}$ as given in (25)), and $\left[\hat{f} ; \hat{f}^{\prime}\right]$ replacing $\hat{f}$ in the RHS. Note that the representation of the interior potential $u^{-}$only involves $A_{0}$, i.e. a non-periodized ring kernel.

In the next section we will demonstrate convergence with respect to the parameters $p$ and $N_{0}$.

Remark 5. Proxy source points have recently been proposed in a similar periodizing scheme for the 3D Laplace equation [39]. However, in App. A we show that for Helmholtz problems spherical harmonics are a much more efficient choice than proxy points.

\subsection{Rapid solution of the linear system}

Since we expect $\mathcal{N}=N P$, the number of columns of $A$, to be $10^{4}$ or greater, the linear system (39) is too large to solve directly (in contrast to related 2D work $[8,9]$ ). Hence we wish to apply an iterative method for the obstacle ring source unknowns $\eta$. We eliminate the smaller number of unknowns in $\xi$, taking a Schur complement of (39), to give

$$
\left(A-B Q^{+} C\right) \eta=\hat{f}
$$

where $Q^{+}$is the Moore-Penrose pseudoinverse of $Q$. This is a rectangular system with poor conditioning similar to that of the axisymmetric MFS system $A_{0} \eta=\hat{f}$ in (26). Note that $A-B Q^{+} C$ computes the one-obstacle interaction $A_{0}$ but with the quasiperiodic Green's function (3); this explains why the scheme breaks down at Wood anomalies. Using (38) and right-preconditioning by $A_{0}^{+}$, we would get the square system

$$
\left(A_{0} A_{0}^{+}+A_{\text {else }} A_{0}^{+}-B Q^{+} C A_{0}^{+}\right) \tilde{\eta}=\hat{f}
$$

from which we can recover the solution $\eta=A_{0}^{+} \tilde{\eta}$. However, since $M>N$ and $A_{0}$ is often rank-deficient in MFS applications, $A_{0}$ has less than full range. $A_{0} A_{0}^{+}$is the orthogonal projector onto the range of $A_{0}$. To create a wellconditioned system which can be solved iteratively, we replace $A_{0} A_{0}^{+}$by the identity, since this has no effect on the resulting desired $\eta$, solving

$$
\left(I+A_{\text {else }} A_{0}^{+}-B Q^{+} C A_{0}^{+}\right) \tilde{\eta}=\hat{f}
$$

via a non-symmetric Krylov method such as GMRES. In effect we are working in a space of surface unknowns $\tilde{\eta}$ rather than ring charge source unknowns $\eta$. Once $\eta$ is known, $\xi=[\mathbf{d} ; \mathbf{a} ; \mathbf{b}]$ is reconstructed via

$$
\xi=-Q^{+} C \eta,
$$

and the solution potential $u$ can then be evaluated anywhere in $\Omega_{\mathrm{box}}$ using $\eta$ and $\mathbf{d}$. The solution in $|z|>z_{0}$ can be evaluated via (9a) using a or (9b) using b. 
(a)

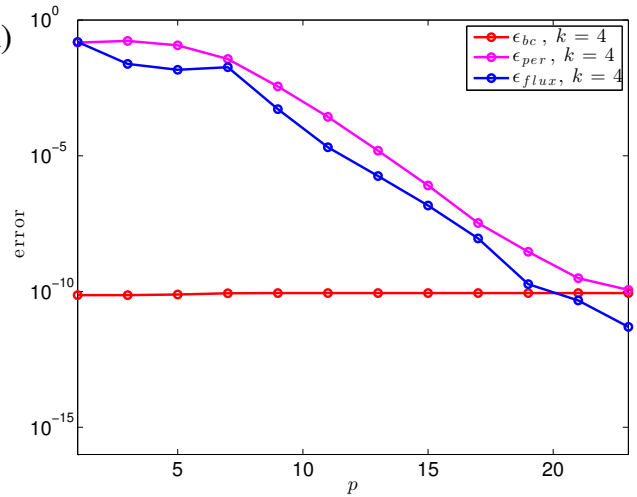

(b)

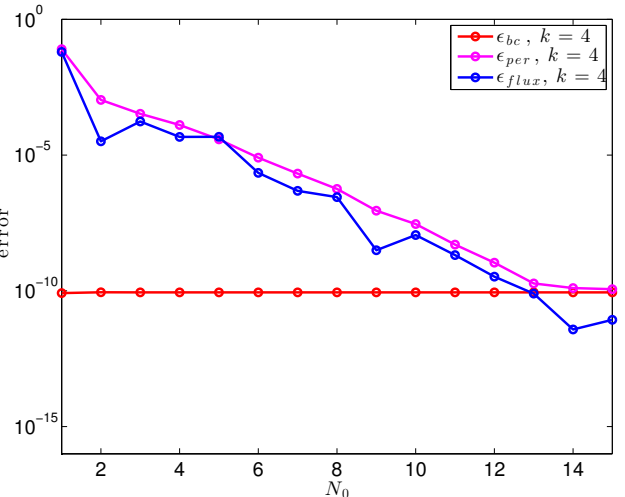

Figure 7: Convergence of errors for the periodizing scheme for the Neumann scattering from a grating of "smooth" objects as in Fig. 2(a), at low frequency. Three types of error are shown: $L_{2}$ norm for the boundary condition, $L_{2}$ norm for the periodicity matching flux conservation, and (a) error vs $p$, fixing $N_{0}=15$; (b) error vs $N_{0}$ when $p=24$.

\begin{tabular}{lcccccccccccccc} 
BVP/shape & $k$ & $N$ & $P$ & $p$ & $N_{0}$ & $M_{1}$ & fill & factor & solve & $\#$ iters & $\epsilon_{\mathrm{bc}}$ & $\epsilon_{\text {per }}$ & $\epsilon_{\text {fux }}$ & RAM \\
\hline \hline Neum/smooth & 4 & 150 & 60 & 24 & 13 & 24 & $22 \mathrm{~s}$ & $4.8 \mathrm{~s}$ & $34 \mathrm{~s}$ & 12 & $9 \mathrm{e}-11$ & $2 \mathrm{e}-10$ & $8 \mathrm{e}-11$ & $2 \mathrm{~GB}$ \\
Trans/smooth & 4,6 & 150 & 60 & 24 & 13 & 24 & $26 \mathrm{~s}$ & $6.6 \mathrm{~s}$ & $36 \mathrm{~s}$ & 12 & $1 \mathrm{e}-11$ & $1 \mathrm{e}-10$ & $2 \mathrm{e}-11$ & $2.5 \mathrm{~GB}$
\end{tabular}

Table 1: Low-frequency periodic scattering example, for the "smooth" shape of Fig. 2(a) in a unit cell $1.3 \lambda$ in period. The columns show wavenumbers $\left(k\right.$, and $k_{-}$when appropriate), numerical parameters, timings, three error metrics, and total RAM usage. The two rows are for Neumann boundary condition, and transmission condition. $N$ is the number of MFS source points, $P$ is the number of Fourier modes, $p$ the maximum degree of the auxiliary spherical harmonic basis, $N_{0}$ the maximum order of the Rayleigh-Bloch expansion. The column "fill" reports the time to fill the matrices, i.e. $A_{0}, B, C$ and $Q$; "factor" reports the factorization time, i.e. doing the SVD on the matrix blocks of $A_{0}$ and on $Q$; while "solve" reports the iterative solver time.

A practical word is needed about handling the pseudoinverses. Because of axisymmetry $A_{0}$ is block diagonal, so each block $A_{n}$ can be inverted independently by taking its SVD to give $A_{n}=U_{n} \Sigma_{n} V_{n}^{*}$. Care must be taken to apply each $A_{n}^{+}$correctly, otherwise a large loss of accuracy results: to compute $A_{n}^{+} x$ for some $x \in \mathbb{C}^{M}$, one uses $V_{n} \Sigma_{n}^{+}\left(U_{n}^{*} x\right)$. The truncation parameter used in $\Sigma_{n}^{+}$is taken as $10^{-10}$. Since there are $P$ blocks, the precomputation required for $A_{0}^{+}$is $O\left(P N^{3}\right)$, then each application takes $O\left(P N^{2}\right)$ with a very small constant. Note that filling $A_{n}^{+}$then using it for matrix-vector multiplication would be dangerous since it is not backward stable; $A_{n}^{+}$may have exponentially large elements which induce catastrophic round-off error (see comments in [57, Sec. 5] and [21, Sec. 3.2]).

Similar care is needed for $Q$ : a dense SVD gives $Q=U \Sigma V^{*}$. Assuming that $y=C A_{0}^{+} \eta$ has already been computed as above, $Q^{+} y$ computed as $V \Sigma^{+}\left(U^{*} y\right)$. The SVD of $Q$ takes $O\left(M_{1}^{2} N_{Q}^{2}\right)$ time. Fixing the obstacle, the numerical parameters $N, P, p, N_{0}$, and $M_{1}$ all grow as $O(k)$. Thus the time for the SVD of $Q$ grows rapidly with wavenumber as $O\left(k^{6}\right)$. This limits the largest $k$ in practice on a standard workstation to one in which the unit cell is around a dozen wavelengths in period.

Remark 6. There are other fast solution methods for the linear system (39). For instance, one could instead eliminate $\eta$ if a fast application of $A^{-1}$ were available (this is done in the analogous $2 D$ periodic scattering problem in [34]).

\begin{tabular}{lcccccccccccccc} 
BVP/shape & $k$ & $N$ & $P$ & $p$ & $N_{0}$ & $M_{1}$ & fill & factor & solve & $\#$ iters & $\epsilon_{\mathrm{bc}}$ & $\epsilon_{\text {per }}$ & $\epsilon_{\text {flux }}$ & RAM \\
\hline \hline Neum/cup & 30 & 240 & 150 & 70 & 21 & 38 & $167 \mathrm{~s}$ & $297 \mathrm{~s}$ & $346 \mathrm{~s}$ & 57 & $2 \mathrm{e}-10$ & $5 \mathrm{e}-11$ & $4 \mathrm{e}-11$ & $17 \mathrm{~GB}$ \\
Neum/cup & 40 & 360 & 180 & 86 & 25 & 45 & $420 \mathrm{~s}$ & $871 \mathrm{~s}$ & $736 \mathrm{~s}$ & 65 & $2 \mathrm{e}-10$ & $2 \mathrm{e}-10$ & $4 \mathrm{e}-11$ & $41 \mathrm{~GB}$ \\
Trans/wiggly & 30,40 & 360 & 200 & 70 & 21 & 38 & $440 \mathrm{~s}$ & $322 \mathrm{~s}$ & $756 \mathrm{~s}$ & 62 & $7 \mathrm{e}-10$ & $4 \mathrm{e}-11$ & $9 \mathrm{e}-11$ & $58 \mathrm{~GB}$ \\
Trans/wiggly & 40,60 & 400 & 200 & 86 & 25 & 45 & $700 \mathrm{~s}$ & $934 \mathrm{~s}$ & $1090 \mathrm{~s}$ & 65 & $1 \mathrm{e}-10$ & $2 \mathrm{e}-10$ & $1 \mathrm{e}-11$ & $93 \mathrm{~GB}$
\end{tabular}

Table 2: Higher-frequency periodic scattering examples (10 $\lambda$ and $13 \lambda$ in period). The first two rows use Neumann boundary conditions on the resonant cup shape, and $\tau=0.03$; the last two rows are for transmission conditions on the "wiggly" shape of Fig. 2(b) and $\tau=0.1$. Other notation is as in Table 1 . 
(a)

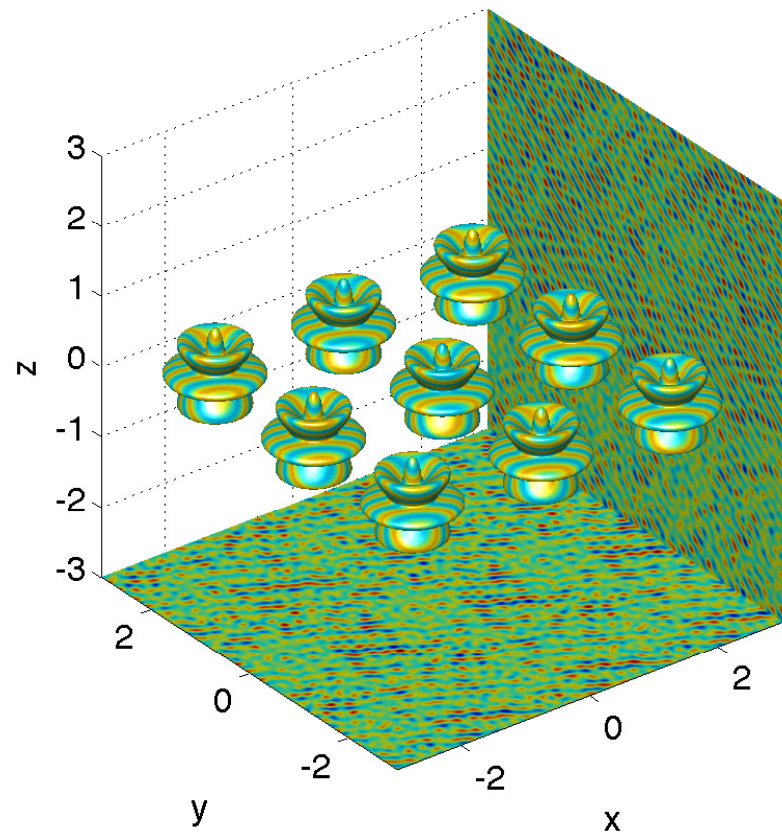

(b)

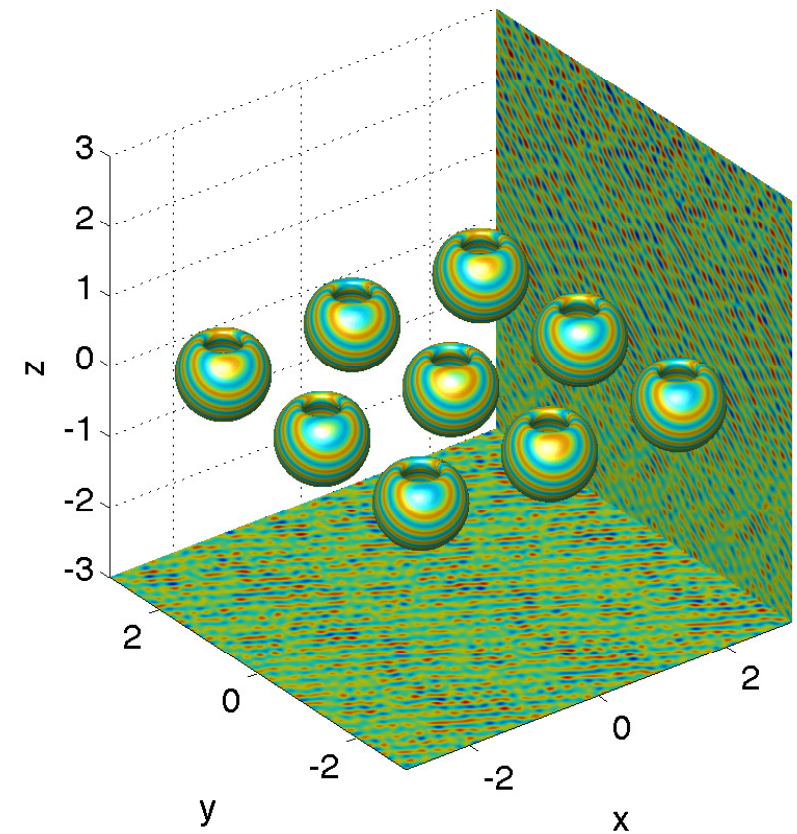

Figure 8: (a) High-frequency periodic transmission scattering solution with $k=40, k_{-}=60$, for the "wiggly" shape of Fig. 2(b). (b) High-frequency periodic Neumann scattering solution with $k=40$, for the cup shape. In both cases the full wave $u^{\mathrm{t}}$ is shown on two slices, the incident wave $u^{\mathrm{i}}$ shown on the obstacle surfaces, and the periodicity is $13 \lambda$. The direction of the incident wave is $\theta=-\frac{\pi}{4}, \phi=\frac{\pi}{3}$, in spherical coordinates.

\section{Results}

We first present some details of our implementation. We use MATLAB R2013b on a desktop workstation with two quad-core E5-2643 CPUs and 128 GB of RAM. The tolerance for GMRES (MATLAB's implementation) is set to $10^{-12}$. We apply $A_{\text {else }}$ via the FMM, using MEX interfaces to the Fortran implementation by Gimbutas-Greengard [35], with the following modification: we set the internal parameter maxlevel=3 in the routine d3tstrcr, which has the effect of limiting the depth of building the oct-tree to 2. Since all sources are well-separated from all targets in $A_{\text {else }}$, this bypasses time spent propagating the source multipoles up the tree via M2M, and local expansions L2L down the tree to the targets. The result is a factor 1-3 speed-up over the vanilla FMM call from this library. We set iprec=3 which requests 9 digits of accuracy in the FMM. Other spherical harmonic evaluations required for $B, C$ and $Q$ are done using a MEX interface to the recurrence relation based fortran libraries in [35], available at http://math.dartmouth.edu/ ahb/software/localexp3d.tgz

The set of MATLAB codes we developed for the tests in this paper will be available at https://math.dartmouth.edu/ yliu/software/acper.tgz

Remark 7. For the $\mathcal{N}$ we test in this work, the dense matrix blocks $B$ and $C$ fit in RAM, and thus we fill them once then apply then via standard BLAS2 matrix-vector multiplies. If they cannot fit in RAM, then they can be applied on the fly using the FMM with only a constant factor change in effort. The (smaller) $Q$ matrix must be stored and inverted densely in our scheme.

Errors for the periodic scattering problem are measured in three different ways:

- $\epsilon_{\mathrm{bc}}$ : an estimate in the $L^{2}$ error in satisfying the boundary condition on $\partial \Omega$ (defined as $\epsilon_{1}$ was in Sec. 3),

- $\epsilon_{\mathrm{per}}$ : an estimate of the $L^{2}$ error in satisfying the periodic boundary conditions on the unit cell walls $L, R, F$ and $B$, and 
- $\epsilon_{\text {flux }}$ : the flux error giving the absolute value of the difference between incoming and outgoing fluxes,

$$
\epsilon_{\text {flux }}:=\left|\sum_{\kappa_{z}^{(m, n)}>0} \kappa_{z}^{(m, n)}\left(\left|a_{m n}\right|^{2}+\left|b_{m n}+e^{-i \kappa_{z}^{(0,0)} z 0} \delta_{m 0} \delta_{n 0}\right|^{2}\right)-\kappa_{z}^{(0,0)}\right|
$$

Note that the incident wave corresponds to Bragg orders $m=n=0$, and the phase shift is needed because the reference for $b_{m n}$ is at $z=-z_{0}$. For the Neumann or transmission BVPs with real-valued $k$ and $k_{-}$(non-absorbing materials), this error should be zero.

There are several numerical parameters, but they fall into two categories. The one-body solution method is controlled by $N, M, P, \tau$, and $q$ (convergence with respect to these being shown in Sec. 3), whereas the periodizing scheme is controlled by $p, N_{0}$, and $M_{1}$ (the latter being fixed at $4 k / \pi$ as explained in Sec. 4.1). We thus first test convergence with respect to the periodizing parameters $p$ and $N_{0}$. Fig. 7 shows error convergence consistent with exponential, in the scattering from a grating of "smooth" obstacles from Fig. 2(a) at low frequency. Note that $\epsilon_{\mathrm{bc}}$ is small throughout the parameter range, and thus cannot alone be used to verify that the full periodic solution has converged. Choosing the converged parameters $p=24$ and $N_{0}=13$, the timing and error results are then given in Table 1, for the two types of BVP. There are $\mathcal{N}=N P=9000$ obstacle unknowns, at the borderline where the FMM starts to become useful. Here the size of $Q$ is 3200 by 1387, making it rapid to invert (via SVD). Thus at low frequency, the computation is dominated by the FMM application of $A_{\text {else }}$ needed in each GMRES iteration.

We show error and timing results at higher frequencies in Table 2 . The wavenumber $k=40$, i.e. a unit cell period of $13 \lambda \times 13 \lambda$, is around the largest that is practical on a single workstation, needing many minutes to take the SVD of the $Q$ matrix of size 16200 by 11493 . The SVD (factorization) and GMRES (solve) stages now take comparable times. The solution (total wave $u^{t}=u+u^{\mathrm{i}}$ ) for the two highest frequency cases are shown on planes in Fig. 8. For evaluation of $u$ we used the FMM for the first term in (35) and direct spherical harmonic summation for the second. Note that the number of GMRES iterations is similar (around 60) for the transmission case as for the highly-resonant Neumann cup shape. The latter models a grating of acoustic Helmholtz resonators. This illustrates that, because the one-obstacle problem is factorized, the iterative part of the scheme is immune to obstacle resonances, in contrast to the case where GMRES is used to solve the entire scattering problem.

\section{Conclusions}

We have presented an acoustic solver for doubly-periodic gratings of smooth axisymmetric obstacles, that is spectrally accurate with respect to all of its convergence parameters, enabling high (10-digit) accuracies to be reached efficiently even at medium to high frequencies. It combines an existing axisymmetric one-body solution using the method of fundamental solutions (MFS) with a new periodizing scheme based on auxiliary spherical harmonics, upward and downward radiation expansions, and collocation on the walls of one unit cell. The result avoids any singular surface quadratures, is efficient for grating periods up to a dozen wavelengths, handles highly resonant obstacles without extra cost, and is relatively simple to code. The one-body factorization cost is $O\left(P N^{3}\right)$ with a small prefactor; in the high-frequency limit this would scale as $O\left(k^{4}\right)$. However, the solve cost per new incident wave is then dominated by only the $O(N P)$ linear cost of an FMM call, i.e. $O\left(k^{2}\right)$. A key feature is that the periodization scheme is independent of the one-body solver, so that the latter could be replaced by an existing boundary integral based solver $[14,15,17]$ without modification, allowing the handling of more general shapes, corners, and edges.

Although our main contribution is the new periodizing scheme, we also have contributed improvements to the one-body MFS scheme. Firstly, we use boundary complexification to place the MFS sources; the choice of distance parameter $\tau$ may be made cheaply using simulations via the analogous 2D BVP. Secondly we give a rigorous analysis of the use of the periodic trapezoid rule for evaluating Helmholtz ring kernels. Unlike in boundary integral equation methods (which require arbitrarily close source-target evaluations), all of our kernels can be evaluated in this way because of the source separation in the MFS. Since applying $\left(A-B Q^{+} C\right)$ is equivalent to applying the quasiperiodic Green's function, our method as presented cannot work at Wood anomalies. However, because the Wood singularity is of inverse square-root form, the neighborhood around which a Wood anomaly causes a loss of accuracy is small, and this accuracy loss is consistent with backwards stability given the accuracy with which the incident wavevector is specified. We note that, were the solver to be extended to handle connected interfaces, robust handling Wood anomalies would then come for free (as in [21]). 


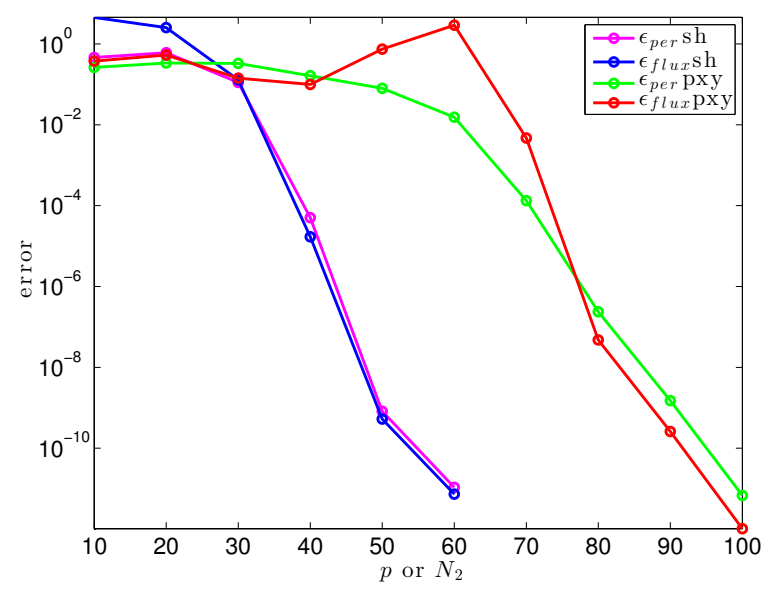

Figure 9: Convergence comparison of spherical harmonics ("sh") vs proxy points ("pxy") as the auxiliary basis for the periodizing scheme, for the Neumann scattering problem from a lattice of "smooth" obstacles as in Fig. 2(a), at wavenumber $k=20$. Note that the two schemes have nearly identical numbers of unknowns when $p=N_{2}$. We have fixed the Rayleigh-Bloch degree as $N_{0}=17$.

Future extensions that would increase the range of application of the solver include: automating the choice of all convergence parameters given a shape and wavenumber $k$; including MFS source point choices that handle axisymmetric edges [60]; generalizing to multilayer media, as in [21]; replacing the eight-neighbor FMM call by a low-rank compression scheme for applying the $A_{\text {else }}$ interactions, which currently dominate the solution time [60]; extension to the Maxwell equations [60]; and replacing the MFS scheme by a boundary integral solver, such as the recent $O\left(\mathcal{N}^{3 / 2}\right)$ fast direct solver [14], to create a 3D version of [33].

\section{Acknowledgments}

The authors thank Fridon Shubitidze for advice about the MFS, Arvind Saibaba for his suggestion for changing from left- to right-preconditioning with the GMRES, and Leslie Greengard and Zydrunas Gimbutas for helpful discussions about tuning the FMM. We also were helped by the comments of the anonymous referees. We are grateful for funding by the National Science Foundation under grant DMS-1216656, and by the Neukom Institute at Dartmouth College.

\section{A. Comparing spherical harmonics vs proxy points as a periodizing basis}

Our periodizing scheme represents the contribution of the lattice of distant copies of the obstacle to the potential with a basis expansion for regular Helmholtz solutions in the unit cell. Here we compare two choices of this basis: the $(p+1)^{2}$ spherical harmonics up to degree $p$ (as used in the rest of this work), vs $N_{2}^{2}$ proxy points placed along lines of longitude on a distant sphere of radius $R$. The latter has been proposed in the context of periodizing the 3D Laplace equation by Gumerov-Duraiswami [39]. The radius $R$ used is $R=3.5$, chosen to optimize proxy point efficiency. The errors we test are $\epsilon_{\text {per }}$ and $\epsilon_{\text {flux }}$ defined in Sec. 5, for the Neumann scattering from a grating of "smooth" obstacles from Fig. 2(a) at wavenumber $k=20$. The period is around $7 \lambda$. We show in Fig. 9 the convergence results, and see that $N_{2} \approx 2 p$ is needed to achieve similar errors in the two representations, i.e. the proxy scheme requires four times the number of unknowns required by the spherical harmonic scheme. (Note that efficiency differences due to the different proxy point arrangements discussed in [39] are small compared to this factor.) We also checked that the solution potential $u$ at the point $(0.9,0.9,0.9)$ agreed between the two methods at their converged parameters with a relative error of $1.2 \times 10^{-11}$. This is consistent with the errors shown in the figure, and provides some verification of the correctness of each scheme. In terms of speed, for comparable 12-digit errors, the SVD of the $Q$ matrix for $p=58$ spherical harmonics takes $217 \mathrm{~s}$, whereas using $N_{2}=100$ proxy points takes $1097 \mathrm{~s}$, around five times slower. Thus we claim that spherical harmonics are by far the better choice for periodizing Helmholtz problems. 


\section{References}

[1] A. Anand, J. S. Ovall, and C. Turc, Well-conditioned boundary integral equations for two-dimensional sound-hard scattering problems in domains with corners, J. Integral Equations Appl. 24 (2012), no. 3, 321-358.

[2] T. Arens, Scattering by biperiodic layered media: The integral equation approach, Ph.D. thesis, Karlsruhe Institute of Technology, 2010.

[3] T. Arens, K. Sandfort, S. Schmitt, and A. Lechleiter, Analysing Ewald's method for the evaluation of Green's functions for periodic media, IMA J. Numer. Anal. 78 (2013), no. 3, 405-431.

[4] H. A. Atwater and A. Polman, Plasmonics for improved photovoltaic devices, Nature Materials 9 (2010), no. 3, 205-213.

[5] I. M. Babuska and S. A. Sauter, Is the pollution effect of the FEM avoidable for the Helmholtz equation considering high wave numbers?, SIAM J. Numer. Anal. 34 (1997), no. 6, 2392-2423.

[6] G. Bao, Finite element approximation of time harmonic waves in periodic structures, SIAM J. Numer. Anal. 32 (1995), no. 4 , $1155-1169$.

[7] A. H. Barnett and T. Betcke, Stability and convergence of the Method of Fundamental Solutions for Helmholtz problems on analytic domains, J. Comput. Phys. 227 (2008), no. 14, 7003-7026.

[8] A. H. Barnett and L. Greengard, A new integral representation for quasi-periodic fields and its application to two-dimensional band structure calculations, J. Comput. Phys. 229 (2010), 6898-6914.

[9] _ A new integral representation for quasi-periodic scattering problems in two dimensions, BIT Numer. Math. 51 (2011), no. 1, 67-90.

[10] A. H. Barnett, L. Greengard, and Z. Gimbutas, Efficient and robust integral equation methods for acoustic scattering from doubly-periodic media in three dimensions, 2015, in preparation.

[11] H Bériot, A Prinn, and G Gabard, Efficient implementation of high-order finite elements for Helmholtz problems, Int. J. Numer. Meth. Engng 106 (2016), 213-240.

[12] A. Bijamov, F. Shubitidze, P. M. Oliver, and D. V. Vezenov, Optical response of magnetic fluorescent microspheres used for force spectroscopy in the evanescent field, Langmuir 26 (2010), 12003-12011.

[13] A.-S. Bonnet-BenDhia and F. Starling, Guided waves by electromagnetic gratings and non-uniqueness examples for the diffraction problem, Math. Meth. Appl. Sci. 17 (1994), 305-338.

[14] J. Bremer, A. Gillman, and P.-G. Martinsson, A high-order accurate accelerated direct solver for acoustic scattering from surfaces, BIT Numer. Math. 55 (2015), no. 2, 367-397.

[15] J. Bremer and Z. Gimbutas, A Nyström method for weakly singular integral operators on surfaces, J. Comput. Phys. 231 (2012), $4885-4903$.

[16] O. P. Bruno and M. C. Haslam, Efficient high-order evaluation of scattering by periodic surfaces: deep gratings, high frequencies, and glancing incidences, J. Opt. Soc. Am. A 26 (2009), no. 3, 658-668.

[17] O. P. Bruno and L. A. Kunyansky, Surface scattering in three dimensions:, Proc. R. Soc. Lond. A 457 (2001), 2921-2934.

[18] O. P. Bruno, S. Shipman, C. Turc, and S. Venakides, Efficient evaluation of doubly periodic Green functions in $3 D$ scattering, including Wood anomaly frequencies, 2013, preprint, arXiv:1307.1176v1.

[19] W. Chen, J. Lin, and C. S. Chen, the method of fundamental solutions for solving exterior axisymmetric Helmholtz problems with high wave-number, Adv. Appl. Math. Mech. 5 (2013), no. 4, 477-493.

[20] H. Cheng, W. Y. Crutchfield, Z. Gimbutas, L. Greengard, F. Ethridge, J. Huang, V. Rokhlin, N. Yarvin, and J. Zhao, A wideband fast multipole method for the Helmholtz equation in three dimensions, J. Comput. Phys. 216 (2006), 300-325.

[21] M. H. Cho and A. H. Barnett, Robust fast direct integral equation solver for quasi-periodic scattering problems with a large number of layers, Opt. Express 23 (2015), no. 2, 1775-1799.

[22] D. Colton and R. Kress, Integral equation methods in scattering theory, Wiley, 1983.

[23] __ Inverse acoustic and electromagnetic scattering theory, second ed., Applied Mathematical Sciences, vol. 93, Springer-Verlag, Berlin, 1998.

[24] J. T. Conway and H. S. Cohl, Exact fourier expansion in cylindrical coordinates for the three-dimensional Helmholtz, Green's function, Z. Angew. Math. Phys. 61 (2010), 425-442.

[25] R. V. Craster and S. Guenneau, Acoustic metamaterials: Negative refraction, imaging, lensing and cloaking, Springer Series in Materials Science (166), Springer, 2013.

[26] P. J. Davis, On the numerical integration of periodic analytic functions, Proceedings of a Symposium on Numerical Approximations (R. E. Langer, ed.), University of Wisconsin Press, 1959.

[27] D. C. Dobson, Optimal design of periodic antireflective structures for the Helmholtz equation, Euro. J. Appl. Math. 4 (1993), $321-340$.

[28] A. Doicu, Y. A. Eremin, and T. Wriedt, Acoustic and electromagnetic scattering analysis using discrete sources, Academic Press, San Diego, CA, 2000.

[29] F. A. Everest and K. C. Pohlmann, Mast handbook for acoustics, 5th ed., McGraw-Hill, 2009.

[30] P. P. Ewald, Die berechnung optischer und elektrostatischer gitterpotentiale, Ann. Phys. 64 (1921), $253-287$.

[31] G. Fairweather and A. Karageorghis, The method of fundamental solutions for elliptic boundary value problems, Adv. Comput. Math. 9 (1998), no. 1-2, 69-95.

[32] K.-Y. Fung and H. Ju, Time domain impedance boundary conditions, Int. J. Comput. Fluid D. 18 (2004), no. 6, $503-511$.

[33] A. Gillman and A. H. Barnett, A fast direct solver for quasiperiodic scattering problems, J. Comput. Phys. 248 (2013), 309-322.

[34] _ _ A fast direct solver for quasiperiodic scattering problems, J. Comput. Phys. 248 (2013), 309-322.

[35] Z. Gimbutas and L. Greengard, FMMLIB3D, Fortran libraries for fast multipole method in three dimensions, 2012, http://www.cims.nyu.edu/cmcl/fmm3dlib/fmm3dlib.html.

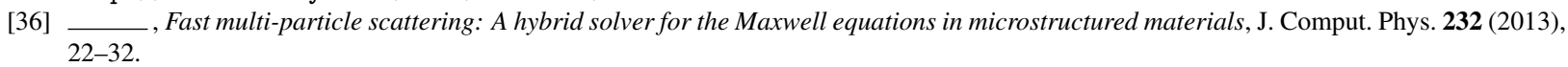

[37] L. Greengard and V. Rokhlin, A fast algorithm for particle simulations, Journal of Computational Physics 135 (1997), no. 2, $280-292$.

[38] N. A. Gumerov and R. Duraiswami, Computation of scattering from clusters of spheres using the fast multipole method, J. Acoust. Soc. Am. 117 (2005), no. 4, 1744-1761.

[39] _ A method to compute periodic sums, J. Comput. Phys. 272 (2014), 307-326 
[40] J. Häggblad and B. Engquist, Consistent modeling of boundaries in acoustic finite-difference time-domain simulations, J. Acoust. Soc. Am. 132 (2012), no. 3, 1303-1310.

[41] S. Hao, P.-G. Martinsson, and P. Young, An efficient and highly accurate solver for multi-body acoustic scattering problems involving rotationally symmetric scatterers, Comput. Math. Appl. 69 (2015), no. 4, 304-318.

[42] J. Helsing and A. Karlsson, An explicit kernel-split panel-based Nyström scheme for integral equations on axially symmetric surfaces, J. Comput. Phys. 272 (2014), 686-703.

[43] _ Determination of normalized magnetic eigenfields in microwave cavities, IEEE Trans. Microw. Theory Tech. 63 (2015), $1457-1467$.

[44] A. Hochman, Y. Leviatan, and J. K. White, On the use of rational-function fitting methods for the solution of $2 D$ Laplace boundary-value problems, J. Comput. Phys. 238 (2013), 337-358.

[45] J. D. Joannopoulos, S. G. Johnson, R. D. Meade, and J. N. Winn, Photonic crystals: Molding the flow of light, 2nd ed., Princeton Univ. Press, Princeton, NJ, 2008.

[46] K. E. Jordan, G. R. Richter, and P. Sheng, An efficient numerical evaluation of the Green's function for the Helmholtz operator on periodic structures, J. Comput. Phys. 63 (1986), 222-235.

[47] R. E. Jorgenson and R. Mittra, Efficient calculation of the free space periodic Green's function, IEEE Trans. Antennas Propagat. 38 (1990), 633-642.

[48] U. Kangro, Convergence of collocation method with delta functions for integral equations of first kind, Integr. Equ. Oper. Theory 66 (2010), no. 2, 265-282.

[49] __ Solution of three-dimensional electromagnetic scattering problems by interior source methods, AIP Conf. Proc. 1479 (2012), 23282331.

[50] A. Karageorghis and G. Fairweather, The method of fundamental solutions for axisymmetric acoustic scattering and radiation problems, J. Acoust. Soc. Am. 104 (1998), no. 6, 3212-3218.

[51] M. Katsurada, Asymptotic error analysis of the charge simulation method in a Jordan region with an analytic boundary, J. Fac. Sci. Univ. Tokyo Sect. IA Math. 37 (1990), no. 3, 635-657. MR MR1080874 (91m:65263)

[52] M. Katsurada and H. Okamoto, A mathematical study of the charge simulation method I, J. Fac. Sci. Univ. Tokyo 35 (1988), $507-518$.

[53] R Kress, On the numerical solution of a hypersingular integral equation in scattering theory, J. Comput. Appl. Math. 61 (1995), 345-360.

[54] R. Kress, Linear integral equations, second ed., Appl. Math. Sci., vol. 82, Springer, 1999.

[55] V. D. Kupradze and M. A. Aleksidze, The method of functional equations for the approximate solution of certain boundary value problems, Comput. Math. Math. Phys. 4 (1964), no. 4, $82-126$.

[56] A. G. Kyurkchan, B. Y. Sternin, and V. E. Shatalov, Singularities of continuation of wave fields, Physics - Uspekhi 12 (1996), $1221-1242$.

[57] J. Lai, M. Kobayashi, and A. H. Barnett, A fast and robust solver for the scattering from a layered periodic structure with multi-particle inclusions, 2014, arXiv: 1412.7466, in review, J. Comput. Phys.

[58] C. M. Linton, Lattice sums for the Helmholtz equation, SIAM Review 52 (2010), no. 4, 603-674.

[59] C. M. Linton and I. Thompson, Resonant effects in scattering by periodic arrays, Wave Motion 44 (2007), 165-175.

[60] Y. Liu, The numerical solution of frequency-domain acoustic and electromagnetic periodic scattering problems, 2016, Ph.D. thesis, Dartmouth College. Available at https://math.dartmouth.edu/ yliu/thesis_YuxiangLiu.pdf.

[61] A. Malcolm and D. P. Nicholls, Operator expansions and constrained quadratic optimization for interface reconstruction: Impenetrable periodic acoustic media, Wave Motion 51 (2014), 23-40.

[62] A. Meier, T. Arens, S. N Chandler-Wilde, and A. Kirsch, A Nyström method for a class of integral equations on the real line with applications to scattering by diffraction gratings and rough surfaces, J. Integral Equations Appl. 12 (2000), 281-321.

[63] R. Model, A. Rathsfeld, H. Gross, M. Wurm, and B. Bodermann, A scatterometry inverse problem in optical mask metrology, J. Phys.: Conf. Ser. 135 (2008), 012071.

[64] M. Möser, Engineering acoustics: An introduction to noise control, Springer-Verlag, 2004.

[65] B. Nennig, E. Perrey-Debain, and J.-D. Chazot, The method of fundamental solutions for acoustic wave scattering by a single and a periodic array of poroelastic scatterers, Eng. Anal. Boundary Elements 35 (2011), 1019-1028.

[66] M. J. Nicholas, A higher order numerical method for 3-D doubly periodic electromagnetic scattering problems, Commun. Math. Sci. 6 (2008), no. 3, 669-694.

[67] Y. Otani and N. Nishimura, A periodic FMM for Maxwell's equations in 3D and its applications to problems related to photonic crystals, J. Comput. Phys. 227 (2008), 4630-52.

[68] Y. Saad and M. H. Schultz, GMRES: a generalized minimal residual algorithm for solving nonsymmetric linear systems, SIAM J. Stat. Sci. Comput. 7 (1986), no. 3, 856-869.

[69] S. Shipman, Resonant scattering by open periodic waveguides, Progress in Computational Physics (PiCP), vol. 1, pp. 7-50, Bentham Science Publishers, 2010.

[70] F. Shubitidze, K. O'Neill, S. A. Haider, K. Sun, and K. D. Paulsen, Application of the method of auxiliary sources to the wide-band electromagnetic induction problem, IEEE Trans. Geosci. Remote Sensing 40 (2002), 928-942.

[71] A. Taflove, Computational electrodynamics: The finite-difference time-domain method, Artech House, Norwood, MA, 1995.

[72] L. N. Trefethen and J. A. C. Weideman, The exponentially convergent Trapezoidal rule, SIAM Review 56 (2014), no. 3, 385-458.

[73] L. Ying, G. Biros, and D. Zorin, A high-order 3D boundary integral equation solver for elliptic PDEs in smooth domains, J. Comput. Phys. 216 (2006), 247-275.

[74] P. Young, S. Hao, and P.-G. Martinsson, A high-order Nyström discretization scheme for boundary integral equations defined on rotationally symmetric surfaces, J. Comput. Phys. 231 (2012), no. 11, 4142-4159.

[75] S. Zhang, C. Xia, and N. Fang, Broadband acoustic cloak for ultrasound waves, Phys. Rev. Lett. 106 (2011), 024301. 